Article

\title{
Vitamin D-Related Genes, Blood Vitamin D Levels and Colorectal Cancer Risk in Western European Populations
}

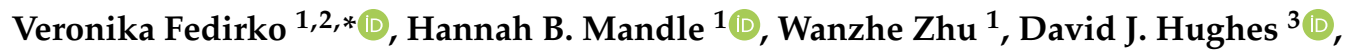
Afshan Siddiq ${ }^{4,5}$, Pietro Ferrari ${ }^{6}$, Isabelle Romieu ${ }^{6}$, Elio Riboli ${ }^{7}$, Bas Bueno-de-Mesquita ${ }^{8}$, Fränzel J.B. van Duijnhoven ${ }^{8}$, Peter D. Siersema ${ }^{9}$, Anne Tjønneland ${ }^{10}$, Anja Olsen ${ }^{10} \mathbb{D}$, Vittorio Perduca 11,12,13 (D), Franck Carbonnel 12,13,14, Marie-Christine Boutron-Ruault 12,13, Tilman Kühn ${ }^{15}$, Theron Johnson ${ }^{15}$, Aleksandrova Krasimira ${ }^{16}$, Antonia Trichopoulou ${ }^{17}$, Periklis Makrythanasis ${ }^{17,18}$, Dimitris Thanos ${ }^{17,18}$, Salvatore Panico ${ }^{19}$, Vittorio Krogh ${ }^{20} \mathbb{D}$, Carlotta Sacerdote 21 (D), Guri Skeie ${ }^{22}$, Elisabete Weiderpass 22,23,24,25,26, Sandra Colorado-Yohar 27,28,29, Núria Sala ${ }^{30}$, Aurelio Barricarte ${ }^{28,31}$, Maria-Jose Sanchez ${ }^{28,32}$, Ramón Quirós ${ }^{33}$, Pilar Amiano 28,34, Björn Gylling ${ }^{35}$, Sophia Harlid ${ }^{36}$, Aurora Perez-Cornago ${ }^{37}$, Alicia K. Heath ${ }^{7}$, Konstantinos K. Tsilidis ${ }^{7,38}$, Dagfinn Aune ${ }^{7,39,40}$, Heinz Freisling ${ }^{6} \mathbb{D}_{\text {, }}$ Neil Murphy ${ }^{6}$, Marc J. Gunter ${ }^{6}$ and Mazda Jenab ${ }^{6, *}$ Lyon 69372, France

Department of Epidemio

8 Division of Human Nutrition \& Health, Wageningen University \& Research, 6700 AA Wageningen, The Netherlands

9 Department of Gastroenterology and Hepatology, Radboud University Medical Center, 6525 GA Nijmegen, The Netherlands

10 Danish Cancer Society Research Center, 2100 Copenhagen, Denmark

11 Laboratoire de Mathématiques Appliquées MAP5, Université Paris Descartes, 75006 Paris, France

12 CESP, Fac. de médecine-Univ. Paris-Sud, Fac. de médecine-UVSQ, INSERM, Université Paris-Saclay, F-94805 Villejuif, France

13 Gustave Roussy, F-94805 Villejuif, France

14 Department of Gastroenterology, Bicêtre University Hospital, Assistance Publique des Hôpitaux de Paris, 94270 Le Kremlin Bicêtre, France

15 Division of Cancer Epidemiology, German Cancer Research Center (DKFZ), 69120 Heidelberg, Germany

16 Nutrition, Immunity and Metabolism, Department of Epidemiology, German Institute for Human Nutrition Potsdam-Rehbrücke, Arthur-Scheunert Allee, 14558 Nuthetal, Germany

17 Hellenic Health Foundation, 11527 Athens, Greece

18 Biomedical Research Foundation of the Academy of Athens, 11527 Athens, Greece

19 Dipartimento Di Medicina Clinica E Chirurgia, Federico Ii University, 80138 Naples, Italy

20 Epidemiology and Prevention Unit, Fondazione IRCCS Istituto Nazionale dei Tumori, Via Venezian, 20133 Milano, Italy

21 Unit of Cancer Epidemiology, Città della Salute e della Scienza University-Hospital and Center for Cancer Prevention (CPO), 10126 Turin, Italy

22 Department of Community Medicine, Faculty of Health Sciences, University of Tromsø, The Arctic University of Norway, 9019 Tromsø, Norway

23 Department of Research, Cancer Registry of Norway, Institute of Population-Based Cancer Research, N-0304 Oslo, Norway 
24 Department of Medical Epidemiology and Biostatistics, Karolinska Institut, SE-171 77 Stockholm, Sweden

25 Genetic Epidemiology Group, Folkhälsan Research Center and Faculty of Medicine, Helsinki University, Helsinki 00014, Finland

26 International Agency for Research on Cancer (IARC-WHO), Lyon 69372, France

27 Department of Epidemiology, Murcia Regional Health Council, IMIB-Arrixaca, Murcia 30008, Spain

28 CIBER Epidemiology and Public Healh (CIBERESP), Madrid 28029, Spain

29 Research Group on Demography and Health, National Faculty of Public Health, University of Antioquia, Cl. 67 \#\#3-108 Medellín, Colombia

30 Unit of Nutrition and Cancer, Cancer Epidemiology Research Program, and Translational Research Laboratory, Catalan Institute of Oncology (ICO)-IDIBELL, 08908 Barcelona, Spain

31 Navarra Public Health Institute, Pamplona 31008, Spain

32 Escuela Andaluza de Salud Pública, Instituto de Investigación Biosanitaria (ibs.GRANADA), Granada 18012, Spain

33 Public Health Directorate, Asturias 33006, Spain

34 Public Health Division of Gipuzkoa, BioDonostia Research Institute, San Sebastian 20014, Spain

35 Department of Medical Biosciences, Pathology, Umeå University, 90187 Umeå, Sweden

36 Department of Radiation Sciences, Oncology, Umeå University, 90187 Umeå, Sweden

37 Cancer Epidemiology Unit, Nuffield Department of Population Health, University of Oxford, Oxford OX3 7LF, UK

38 Department of Hygiene and Epidemiology, University of Ioannina School of Medicine, Ioannina 45110, Greece

39 Department of Nutrition, Bjørknes University College, 0456 Oslo, Norway

40 Department of Endocrinology, Morbid Obesity and Preventive Medicine, Oslo University Hospital, 0372 Oslo, Norway

* Correspondence: vfedirk@emory.edu (V.F.); jenabm@iarc.fr (M.J.)

Received: 21 June 2019; Accepted: 12 August 2019; Published: 20 August 2019

Abstract: Higher circulating 25-hydroxyvitamin D levels (25(OH)D) have been found to be associated with lower risk for colorectal cancer (CRC) in prospective studies. Whether this association is modified by genetic variation in genes related to vitamin $\mathrm{D}$ metabolism and action has not been well studied in humans. We investigated 1307 functional and tagging single-nucleotide polymorphisms (SNPs; individually, and by gene/pathway) in 86 vitamin D-related genes in 1420 incident CRC cases matched to controls from the European Prospective Investigation into Cancer and Nutrition (EPIC) cohort. We also evaluated the association between these SNPs and circulating $25(\mathrm{OH}) \mathrm{D}$ in a subset of controls. We confirmed previously reported CRC risk associations between SNPs in the VDR, GC, and CYP27B1 genes. We also identified additional associations with $25(\mathrm{OH}) \mathrm{D}$, as well as CRC risk, and several potentially novel SNPs in genes related to vitamin D transport and action ( LRP2, CUBN, NCOA7, and HDAC9). However, none of these SNPs were statistically significant after Benjamini-Hochberg $(\mathrm{BH})$ multiple testing correction. When assessed by a priori defined functional pathways, tumor growth factor $\beta$ (TGF $\beta)$ signaling was associated with $C R C$ risk $(P \leq 0.001)$, with most statistically significant genes being SMAD7 $\left(P_{B H}=0.008\right)$ and SMAD3 $\left(P_{B H}=0.008\right)$, and 18 SNPs in the vitamin $D$ receptor (VDR) binding sites $(P=0.036)$. The $25(\mathrm{OH}) \mathrm{D}$-gene pathway analysis suggested that genetic variants in the genes related to VDR complex formation and transcriptional activity are associated with CRC depending on $25(\mathrm{OH}) \mathrm{D}$ levels (interaction $P=0.041$ ). Additional studies in large populations and consortia, especially with measured circulating $25(\mathrm{OH}) \mathrm{D}$, are needed to confirm our findings.

Keywords: single nucleotide polymorphism (SNP); vitamin D; colorectal neoplasms; incidence 


\section{Introduction}

Colorectal cancer (CRC) is the second most common cancer in men and women combined, with approximately 1.4 million new cases diagnosed in 2012 worldwide [1]. There is compelling observational evidence that low circulating vitamin D concentrations are associated with increased risk of incident CRC [2,3]. However, other human evidence is less convincing. A few Mendelian randomization $(\mathrm{MR})$ studies did not support an association between vitamin $\mathrm{D}$ genetic score and CRC risk, but the genetic contribution to $25(\mathrm{OH}) \mathrm{D}$ is relatively small $(7.5 \%$ as estimated based on genome-wide association studies (GWAS) on common SNPs [4]), possibly explaining the null findings [5,6]. Also, the relatively few randomized clinical trials (RCTs) of vitamin D supplementation and colorectal neoplasms have not shown statistically significant effects, but sample size, duration and timing of supplementation, issues with compliance and choice of study population, and the limited range of vitamin D exposures assessed may have contributed to the null results [7-9]. Finally, the benefits from vitamin D supplementation for the prevention of colorectal neoplasms may vary according to genetic variation in the vitamin D-related genes (e.g., vitamin D receptor (VDR) [10]).

Anti-neoplastic effects of vitamin D on colorectal tissue are also supported by the fact that the normal colorectal epithelium expresses the vitamin D receptor (VDR) and vitamin D metabolizing enzymes (CYP27B1 and CYP24A1) and, therefore, can locally produce and degrade the active form of vitamin $\mathrm{D}, 1$,25-dihydroxyvitamin $\mathrm{D}\left(1,25(\mathrm{OH})_{2} \mathrm{D}\right)$, from 25-hydroxyvitamin $\mathrm{D}(25(\mathrm{OH}) \mathrm{D})$ [11-13]. In the colorectum, the active metabolite of vitamin $\mathrm{D}, 1,25(\mathrm{OH})_{2} \mathrm{D}$, exerts its anti-neoplastic effects by genomic (mediated by the VDR) and non-genomic mechanisms [14], including the regulation of over 200 vitamin D-responsive genes and rapid activation of intracellular signaling pathways, resulting in modulation of the cell cycle, bile acid degradation, immune response, growth factor signaling, and anti-inflammation [15].

Observational and RCT data suggest a potential vitamin D-colorectal neoplasms risk association is modified by polymorphisms in the vitamin D receptor (VDR) $[10,16,17]$ and the vitamin D-binding protein gene (GC) [18]; however, only a few single nucleotide polymorphisms (SNPs) and a limited number of related pathways were considered. Novel evidence highlights a wide array of VDR binding sites across the human genome [19], and multiple pathways related to vitamin D effects [20]. Thus, it is plausible that the vitamin D-CRC risk association may be modulated by variation in a broad array of genes related to vitamin D metabolism (e.g. absorption, endogenous synthesis, transport, activation, and deactivation) and action (including transcriptional activity/post-transcriptional effects). All of these genes are polymorphic, but no studies to date have comprehensively investigated their individual and collective associations with CRC risk or circulating vitamin D levels. In consideration of these points, we investigated whether variation in genes related to vitamin D metabolism and transcriptional activity is related to circulating blood vitamin D levels, and whether genetic variation at the SNP, pathway and gene level, alone and in combination with circulating vitamin D levels, is associated with CRC risk in a large Western European prospective cohort study.

\section{Materials and Methods}

\subsection{Study Population}

We used a case-control design nested within the European Prospective Investigation into Cancer and Nutrition (EPIC) cohort, a large prospective study with over 520,000 men and women aged 35-70 years enrolled from 23 centers in 10 Western European countries (Denmark, France, Greece, Germany, Italy, the Netherlands, Norway, Spain, Sweden, and United Kingdom). The methods of the EPIC study have been described in detail elsewhere [21,22]. Individuals who were eligible for the study were selected from the general population of a specific geographical area, town, or province. Exceptions included the French sub-cohort, which is based on members of the health insurance system or state-school employees, and the Utrecht (Netherlands) sub-cohort, which is based on women who underwent screening for breast cancer. Between 1992 and 1998, standardized lifestyle and personal 
history information, anthropometrics, and blood samples were collected from most participants at recruitment. Diet over the previous 1 year was measured at baseline by validated country-specific dietary questionnaires developed to ensure high compliance and better measures of local dietary habits [21]. Blood samples were stored at the International Agency for Research on Cancer (Lyon, France; $-196{ }^{\circ} \mathrm{C}$, in liquid nitrogen) for all countries except Denmark $\left(-150{ }^{\circ} \mathrm{C}\right.$, in nitrogen vapor) and Sweden (in $-80^{\circ} \mathrm{C}$ freezers). The EPIC study was approved by the Ethical Review Board of the International Agency for Research on Cancer (IARC) and the Institutional Review Board of each participating EPIC center. Written consent was obtained from all EPIC participants at enrolment into the study.

\subsection{Cancer Incidence and Vital Status Follow-Up}

Cancer incidence was determined through record linkages with regional cancer registries (Denmark/Italy/the Netherlands/Norway/Spain/Sweden/United Kingdom; complete up to December 2006) or via a combination of methods, including the use of health insurance records, contacts with cancer and pathology registries, and active follow-up through study subjects and their next-of-kin (France/Germany/Naples/Greece; complete up to June 2010).

Vital status follow-up (98.5\% complete) was collected by record linkage with regional and/or national mortality registries in all countries except France, Germany, and Greece, where data are collected through an active follow-up. Censoring dates for complete follow-up were between June 2005 and June 2009 in Denmark, the Netherlands, Spain, the United Kingdom, Sweden, Norway, and Italy. In Germany, Greece, and France follow-up was based on a combination of methods, including health insurance records, cancer and pathology registries, and active follow-up through study subjects and their next-of-kin. In these centers, the end of follow-up was defined as the last known date of contact, or the date of death whichever came first. The last update of endpoint information occurred between December 2007 and December 2009.

\subsection{Nested Case-Control Design and Participant Selection}

\subsubsection{Case Ascertainment and Selection}

CRC cases were selected among participants who developed colon (C18.0-C18.7, according to the ICD-10), rectum (C19-C20), and overlapping/unspecified origin tumors (C18.8 and C18.9). Cancers of the anus were excluded. CRC is defined as the combination of the colon and rectal cancers.

A total of 1420 first-time previously cancer-free colorectal cancer cases (colon cancer $=900$; rectal cancer $=520$ ) were identified. Cases were not selected from Norway (blood samples only recently collected; few colorectal cancers diagnosed after blood donation) and the Malmö center of Sweden. The number of cases for gene-environment analyses was 1176 because of missing, previously collected 25(OH)D measurements [23] (France $=6$, Italy $=49$, Spain $=30, \mathrm{UK}=27$, The Netherlands $=8$, Greece $=18$, Germany $=21$, and Sweden $=16$ ).

\subsubsection{Control Selection}

Controls were selected (1:1) by incidence density sampling from all cohort members alive and not having a reported cancer at the time of diagnosis of the cases and were matched by age ( \pm 6 months at recruitment), sex, study center, time of the day at blood collection, and fasting status at the time of blood collection (less than three hours, three to six hours, and more than six hours). Women were further matched by menopausal status (pre-/post-/peri-menopausal, and unknown) and for pre-menopausal women, phase of menstrual cycle at time of blood collection and usage of postmenopausal hormone therapy at time of blood collection (yes/no, regardless of menopausal status). The additional matching criteria for women were required for other studies that were being carried out using the same matched case-control sets. One control sample failed the genotyping and was not included in the analysis, resulting in a total of 1419 controls. The number of controls for analyses involving 25(OH)D was 764 
because of missing or unobtainable, previously collected $25(\mathrm{OH}) \mathrm{D}$ measurements [23] (France $=18$, Italy $=69$, Spain $=48, \mathrm{UK}=62$, The Netherlands $=41$, Greece $=23$, Germany $=60$, Sweden $=49$, and Denmark $=328$ ).

\subsubsection{Blood 25-(OH)-Vitamin D Assessment}

We previously measured blood concentrations of $25(\mathrm{OH}) \mathrm{D}$ using a commercially available enzyme immunoassay kit (OCTEIA 25-(OH)D Kit, Immuno Diagnostic Systems, Boldon, UK) at the Laboratory for Health Protection Research, National Institute for Public Health and the Environment, the Netherlands [23]. The kit is specific for $100 \%$ of $25-(\mathrm{OH})$-vitamin $\mathrm{D}_{3}$ form and $75 \%$ of $25-(\mathrm{OH})$-vitamin $\mathrm{D}_{2}$ form. The inter-assay coefficient of variation as determined with two kit control samples was minimal $(5.9 \%$ at the level of $20.3 \mathrm{nmol} / \mathrm{L}$ and $5.4 \%$ at the level of $77.4 \mathrm{nmol} / \mathrm{L})$. No significant between-day drift, time shifts, or other trends were observed and the percentage of variance attributable to batch-to-batch differences was $4.5 \%$. For all analyses, laboratory technicians were blinded to the case-control status of the samples.

\subsubsection{SNP Selection, Genotyping, and Quality Control}

Genomic DNA was extracted from whole blood samples using conventional methods. We used the custom GoldenGate Universal-32 3072-plex assay kit (Illumina, CA, USA) to genotype 1716 genetic variants within the genes known and proposed to be involved in (1) vitamin D metabolism (DHCR7, GC, CYP3A4, CYP2R1, CYP24A1, CUBN, and LRP2), (2) mineral homeostasis and endocrine regulations of $1,25(\mathrm{OH})_{2} \mathrm{D}$ synthesis (CASR, PTH, TRPV5, and TRPV6), (3) vitamin D genomic effects (VDR, $R X R A, R X R B$, and $R X R G)$, (4) formation of the VDR complex (co-activators and co-regulators ACTL6A, ARID1A, BAZ1B, CARM1, CHAF1A, CREBBP, EP300, HDAC9, MED1, NCOA1, NCOA2, NCOA3, NCOA7, NCOR1, NCOR2, PCAF/KAT2B, PRMT1, SMARCA2, SMARCA4, SMARCC1, SMARCD1, SMARCE1, SNW1, SUPT16H, TOP2B, and TSC2), and (5) vitamin D post-transcriptional response (tumor growth factor $\beta$ (TGF $\beta$ )-signaling, inflammation, oxidative stress, insulin growth factor (IGF) signaling, cell cycle, and VDR binding sites; please see Supplementary Table S1 for a complete list of genes and SNPs). The custom GoldenGate assay was designed using the Illumina online Assay Design Tool in May 2012. SNP genotype dataset for CEU population (Utah residents with Northern and Western European ancestry; HapMap Data Rel 28 Phase II + III, August 10, on NCBI B36 assembly, dbSNP b126) were loaded in the Haploview program (Broad Institute, MIT and Harvard, Cambridge, MA, USA) and SNPs with minor allele frequencies (MAFs) greater than $5 \%$ and the $\mathrm{r}^{2}$ linkage disequilibrium (LD) statistic of 0.8 were selected as tagging SNPs (tagSNPs). Additionally, we searched published literature for previously reported functional and regulatory SNPs in the genes of interest and included them in genotyping irrespective of MAFs or $r^{2}$ with other SNPs. Genotyping was performed by the Genetics Laboratory at Imperial College London. After excluding 409 SNPs [247 (14.4\%) that failed genotyping, 54 (3.1\%) that failed to satisfy the Hardy-Weinberg criterion (Supplementary Table S1), 98 $(5.7 \%)$ missing in more than $20 \%$ of genotyped samples, and $10(0.6 \%)$ that were monomorphic], a total of 1307 SNPs were included in the analysis. All genotyping underwent standard quality control including concordance checks for blinded duplicates and examination of sample and SNP call rates. The lowest reproducibility frequency across 62 replicate samples was 0.98 . The call rate was $95 \%$ for all samples and $95 \%$ for all SNPs. 


\subsubsection{Statistical Analysis}

The season adjustment of $25(\mathrm{OH}) \mathrm{D}$ was carried out by the week of blood draw using the sine curve method [24]. The associations between season-adjusted $25(\mathrm{OH}) \mathrm{D}$ concentrations and genetic variants (coded as 0,1, 2 corresponding to the number of minor alleles) were assessed among controls using linear regression models adjusted for age, sex, and center. Further adjustment for BMI, smoking status, and physical activity did not change the results substantially. We used unconditional logistic regression analysis to assess the association of individual SNPs with CRC risk, adjusting for age (continuous), sex, and study center. Results were similar when we used conditional logistic regression on 1331 complete case-matched sets. We assumed a log-additive genetic model, but also tested dominant and recessive models as the underlying genetic model for these SNPs is unknown. Further adjustment for body mass index (BMI; continuous), smoking status (never, former, current smokers, missing), physical activity (active, moderately active, moderately inactive, and inactive), alcohol intake (continuous), hormone therapy, and menopausal status did not substantially change the results, and thus these variables were not included in the final statistical model. Subgroup analyses were conducted by sex and tumor location (colon vs. rectum).

To examine the associations between genes (a combination of SNPs) and genetic pathways (a combination of genes) and CRC risk, we used the Adaptive Rank Truncated Product (ARTP) method [25] as implemented in the first step (no interaction) of the R package PIGE (http://cran. r-project.org/web/packages/PIGE/index.html). This method can combine associations of SNPs in each gene (or from the genes in a pathway) to provide a $P$-value at the gene or pathway level, respectively. Genetic markers in high $\mathrm{LD}\left(\mathrm{r}^{2} \geq 0.8\right)$ were excluded using the AdaJoint $\mathrm{R}$ package (https://cran.r-project.org/web/packages/ARTP2). To investigate the multiplicative interaction between the genes and genetic pathways with $25(\mathrm{OH}) \mathrm{D}$ on CRC risk, we used the modified ARTP method as implemented in the R package PIGE. The $P$-values at the SNP and the gene levels were corrected for multiple testing for the number of SNPs and for the number of genes, respectively, using the false discovery rate (Benjamini-Hochberg or BH) method [26]. Furthermore, we used traditional methods to assess potential interactions between SNPs and 25(OH)D stratifying by categories of $25(\mathrm{OH}) \mathrm{D}$ concentrations and assuming a log-additive model for genetic markers. Also, we assessed the association of $25(\mathrm{OH}) \mathrm{D}$ (per $24.96 \mathrm{nmoL}=10 \mathrm{ng} / \mathrm{mL}$ ) with CRC risk by genotype.

All statistical tests were two-sided with $P$-values $<0.05$ considered statistically significant (SAS software, version 9.2; SAS Institute, Cary/NC; R, R Foundation for Statistical Computing, Vienna/Austria).

\section{Results}

\subsection{Baseline Characteristics of Cases and Controls}

Selected baseline characteristics of the CRC cases and matched controls are shown in Table 1. The mean age at blood donation of cases and controls was 58 years. On average, CRC cases had 4 years between blood donation and the time of diagnosis. The dataset included 520 rectal cancer cases and 900 colon cancer cases. 
Table 1. Selected baseline characteristics of incident colorectal cancer (CRC) cases and their matched controls, the European Prospective Investigation into Cancer and Nutrition (EPIC) study, 1992-2003.

\begin{tabular}{|c|c|c|c|c|}
\hline \multirow[t]{2}{*}{ Baseline Characteristic } & \multicolumn{2}{|c|}{ Cases } & \multicolumn{2}{|c|}{ Controls } \\
\hline & \multicolumn{2}{|c|}{$\mathrm{n}=1420$} & \multicolumn{2}{|c|}{$\mathrm{n}=1419$} \\
\hline Women, N (\%) & 705 & $(49.6)$ & 701 & $(49.4)$ \\
\hline Mean age at blood collection, (SD) years & 58.5 & $(7.3)$ & 58.6 & $(7.3)$ \\
\hline Mean years of follow-up, (SD) years & 4.1 & (2.3) & - & \\
\hline \multicolumn{5}{|l|}{ Smoking status, $\mathrm{N}(\%)^{\mathrm{a}}$} \\
\hline Never & 580 & $(40.8)$ & 594 & $(41.9)$ \\
\hline Former & 476 & $(33.5)$ & 460 & $(32.4)$ \\
\hline Current & 346 & $(24.4)$ & 349 & $(24.6)$ \\
\hline \multicolumn{5}{|l|}{ Physical activity, N (\%) } \\
\hline Inactive & 202 & $(14.2)$ & 183 & $(12.9)$ \\
\hline Moderately inactive & 402 & $(28.3)$ & 367 & $(25.9)$ \\
\hline Moderately active & 583 & $(41.1)$ & 612 & $(43.1)$ \\
\hline Active & 130 & $(9.2)$ & 148 & $(10.4)$ \\
\hline BMI, (SD) kg/m² & 26.8 & $(4.2)$ & 26.3 & $(3.8)$ \\
\hline 25-(OH)-vitamin D measurement, N (\%) & 1,176 & $(82.8)$ & 764 & $(53.8)$ \\
\hline $25-(\mathrm{OH})$-vitamin $\mathrm{D}$, mean $(\mathrm{SD}) \mathrm{nmol} / \mathrm{L}^{\mathrm{b}}$ & 58.5 & $(25.6)$ & 62.0 & $(25.4)$ \\
\hline \multicolumn{5}{|l|}{ Country, N (\%) } \\
\hline France & 28 & $(2.0)$ & 29 & $(2.0)$ \\
\hline Italy & 202 & $(14.2)$ & 198 & $(14.0)$ \\
\hline Spain & 146 & $(10.3)$ & 141 & $(9.9)$ \\
\hline United Kingdom & 240 & $(16.9)$ & 250 & $(17.6)$ \\
\hline The Netherlands & 153 & $(10.8)$ & 158 & (11.1) \\
\hline Greece & 46 & $(3.2)$ & 48 & $(3.4)$ \\
\hline Germany & 179 & (12.6) & 169 & (11.9) \\
\hline Sweden & 88 & $(6.2)$ & 86 & $(6.1)$ \\
\hline Denmark & 338 & $(23.8)$ & 340 & $(24.0)$ \\
\hline
\end{tabular}

a Percent missing is not shown. Therefore the total percentages do not add up to $100 \%$. ${ }^{\mathrm{b}}$ Season standardized using the sine-curve method [25].

\subsection{SNPs in the Genes Related to Vitamin D Metabolism/Transcriptional Activity and 25(OH)D}

Thirty-seven SNPs in the genes related to vitamin D metabolism, formation of the VDR complex, and $V D R$ transcriptional activity were associated with season-adjusted $25(\mathrm{OH}) \mathrm{D}$ concentrations with unadjusted $P \leq 0.05$ among controls (Supplementary Table S2). The top 10 SNPs are shown in Table 2 . Of the 37, 17 SNPs were in the genes involved in vitamin D metabolism, and 20 SNPs in the genes involved in vitamin D transcriptional activity. None of these SNPs were statistically significantly associated with $25(\mathrm{OH}) \mathrm{D}$ after BH correction. The associations of all SNPs with 25(OH)D among controls only are shown in Supplementary Table S3A, and among cases and controls combined in Supplementary Table S3B. 
Table 2. Top 10 single-nucleotide polymorphisms (SNPs) in the genes related to vitamin D metabolism and transcriptional activity associated with season-adjusted $25(\mathrm{OH}) \mathrm{D}$ concentrations among controls only, the EPIC study, 1992-2003 ${ }^{\text {a }}$.

\begin{tabular}{|c|c|c|c|c|c|}
\hline Gene $^{b}$ & SNP & $\mathbf{N}$ & $25(\mathrm{OH}) \mathrm{D}, \beta(95 \% \mathrm{CI})$ & $P$ & $P_{B H}^{c}$ \\
\hline$V D R$ & rs2239182 & 742 & $-3.82(-6.15,-1.49)$ & 0.001 & 0.949 \\
\hline LRP2 & rs2673170 & 747 & $-4.43(-7.27,-1.58)$ & 0.002 & 0.949 \\
\hline NCOA7 & rs579477 & 758 & $-3.57(-6.07,-1.07)$ & 0.005 & 0.949 \\
\hline GC & rs1352844 & 747 & $5.26(1.63,8.88)$ & 0.005 & 0.949 \\
\hline GC & rs188812 & 752 & $5.38(1.57,9.20)$ & 0.006 & 0.949 \\
\hline GC & rs2298849 & 757 & $4.26(1.11,7.41)$ & 0.008 & 0.949 \\
\hline CUBN & rs 4525114 & 750 & $7.11(1.94,12.29)$ & 0.007 & 0.949 \\
\hline CYP27B1 & rs4646536 & 751 & $3.65(0.93,6.37)$ & 0.009 & 0.949 \\
\hline CYP27B1 & rs10877013 & 764 & $3.42(0.73,6.12)$ & 0.013 & 0.974 \\
\hline HDAC9 & rs212669 & 753 & $-8.12(-14.34,-1.90)$ & 0.011 & 0.974 \\
\hline
\end{tabular}

a Adjusted for age at blood collection, sex, and center. ${ }^{\mathrm{b}}$ Genes related to vitamin D metabolism and transcriptional activity. ${ }^{\mathrm{c}} \mathrm{P}$ after Benjamini-Hochberg (BH) multiple testing correction.

\subsection{SNPs in the Genes Related to Vitamin D Metabolism/Function and CRC Risk}

We examined the associations between SNPs in the genes involved in vitamin D metabolism (genes $=9$, SNPs $=274$ ), mineral homeostasis and endocrine regulation of $1,25(\mathrm{OH})_{2} \mathrm{D}$ synthesis (genes $=5$, SNPs $=58$ ), vitamin $\mathrm{D}$ genomic effects including the VDR complex co-activators and co-regulators (genes $=30$, SNPs $=538$ ), and two SNPs in the intergenic regions previously associated with circulating 25(OH)D [27] and CRC risk (Supplementary Table S4). In Table 3, we show the top fifteen statistically significant SNPs associated with CRC risk defined by $P_{\text {unadjusted }}<0.01$. However, after $\mathrm{BH}$ correction, none of the associations remained statistically significant (all $\left.P_{B H}>0.2\right)$. The results did not differ by tumor location (Table 3 and Supplementary Table S4) or sex (Supplementary Table S5).

\subsection{SNPs in the Vitamin D-Responsive Genes and CRC Risk}

We also examined the associations between 434 SNPs in the genes responsive to vitamin D, including the genes in the TGF $\beta$ and IGF signaling pathways, inflammation, oxidative stress, cell cycle, and 19 SNPs located in the VDR binding sites as previously published [19] (Supplementary Table S6). Twenty-five SNPs were significantly associated with CRC risk at $P<0.01$. However, after BH correction, none of the associations (except for SMAD3 rs7180244; SMAD7 rs11874392, rs12953717 and rs4939827) remained statistically significant (Supplementary Table S7). Interestingly, three SNPs (rs3197999, rs3802842, rs762421) in previously identified VDR binding sites were associated with CRC risk. The results did not differ by tumor location (Supplementary Tables S6 and S7) or sex (Supplementary Table S8).

\subsection{Vitamin D Genes/Pathways and CRC Risk}

At the pathway level, the $V D R$ binding sites and TGF $\beta$ signaling pathway were statistically significantly associated with CRC risk $(P<0.04$; Table 4$)$. For colon cancer, in addition to the VDR binding sites $(P=0.008)$ and TGF $\beta$ signaling pathway $(P=0.0001)$, an association with cell cycle pathway was observed $(P=0.03)$. The TGF $\beta(P=0.0001)$ and IGF $(P=0.007)$ signaling pathways, but not the $V D R$ binding sites $(P=0.256)$, were statistically significantly associated with rectal cancer risk.

At the gene level, several genes (CHAF1A, SMARCE1, SMAD7, SMAD3, BMP2, and C-MYC region) were associated with $C R C$ risk at unadjusted $P<0.05$. However, all of them except $S M A D 7\left(P_{B H}=0.008\right)$ and SMAD3 $\left(P_{B H}=0.008\right)$ were not statistically significant after BH correction. The SMAD7, SMAD3, $B M P 2$, and $C-M Y C$ regions were associated with colon cancer; however, after $\mathrm{BH}$ correction, only $S M A D 7\left(P_{B H}=0.04\right)$ and SMAD3 $\left(P_{B H}=0.009\right)$ remained statistically significant. In addition to SMAD7 $(P=0.0005)$ and SMAD3 $(P=0.0003)$, several other genes or genetic regions $(C Y P 2 R 1, C H A F 1 A$, CREBBP, IL10, SNPs identified in genome-wide association studies (GWAS) to be associated with IGF 
levels, IGFBP2/IGFBP5, IGFBP3, and C-MYC region) were associated with rectal cancer. However, after BH correction, only SMAD7 $(P=0.02)$ and SMAD3 $(P=0.02)$ remained statistically significant.

\subsection{5. (OH)D-Gene and 25(OH)D Pathway Interactions and CRC Risk}

At the pathway level, the VDR complex and its transcriptional co-regulators and co-activators demonstrated a potential interaction with $25(\mathrm{OH}) \mathrm{D}$ concentrations in the association with CRC risk $(P=0.04$; Table 4). Within this pathway, the interaction $P$-values of $<0.05$ were observed for $A R I D 1 A$, CARM1, CHAF1A, and SMARCA2, but none were statistically significant after BH correction. Similar associations were observed for colon cancer, but not rectal cancer ( $P$ for interaction for the $V D R$ complex and its transcriptional co-regulators and co-activators were 0.105 and 0.727 , respectively).

At the gene level, the interaction $P$-values of $<0.05$ were observed for CYP27B1 and GC (vitamin D metabolism) and IL10 (inflammation) for CRC and colon cancer. Also, the interaction between 25(OH)D and IGFBP2/IGFBP5 was statistically significant for colon cancer. For rectal cancer, the interaction $P$-values of $<0.05$ were observed for CYP24A1 (vitamin D metabolism) and BMP2 (TGF signaling). None of the gene-25(OH)D interactions were statistically significant after $\mathrm{BH}$ correction.

Next, we assessed the associations between 25(OH)D (per $24.96 \mathrm{nmol} / \mathrm{L}$ ) with CRC risk, stratified by genotypes of SNPs in the genes that were identified in the step above as potentially modifying the association of 25(OH)D with CRC risk (CYP27B1, GC, ARID1A, CARM1, CHAF1A, SMARCA2, and IL10; Supplementary Table S9). Sixteen SNPs in these genes with $P$ for interaction $<0.05$ are presented in Table 5. None were statistically significant after BH correction. Several SNPs had a very low number of minor allele homozygotes, with no effect estimates presented in the table. 
Table 3. Associations of SNPs with CRC risk overall and by tumor location (colon vs. rectum), the EPIC study, 1992-2003.

\begin{tabular}{|c|c|c|c|c|c|c|c|c|c|c|c|c|c|c|}
\hline \multirow{2}{*}{ Gene/SNP } & \multirow{2}{*}{ Genotype } & \multicolumn{5}{|c|}{ Colorectal Cancer } & \multicolumn{4}{|c|}{ Colon Cancer } & \multicolumn{4}{|c|}{ Rectal Cancer } \\
\hline & & Cases & Controls & OR $(95 \% \mathrm{CI})^{\mathrm{a}}$ & $P$ & $P_{B H}$ & Cases & OR $(95 \% \mathrm{CI})^{\mathrm{a}}$ & $P$ & $P_{B H}$ & Cases & OR $(95 \% \mathrm{CI})^{\mathrm{a}}$ & $P$ & $P_{B H}{ }^{b}$ \\
\hline \multirow{7}{*}{$\begin{array}{c}\text { CUBN } \\
\text { rs12243895 }\end{array}$} & & & & & & & & & & & & & & \\
\hline & GG & 702 & 767 & 1.00 (ref) & 0.009 & 0.569 & 435 & 1.00 (ref) & 0.006 & 0.509 & 267 & 1.00 (ref) & 0.261 & 0.969 \\
\hline & GA & 551 & 513 & $1.18(1.00,1.38)$ & & & 354 & $1.20(1.00,1.44)$ & & & 197 & $1.12(0.90,1.40)$ & & \\
\hline & AA & 140 & 104 & $1.48(1.12,1.96)$ & & & 94 & $1.59(1.17,2.16)$ & & & 46 & $1.34(0.91,1.97)$ & & \\
\hline & Additive & 1393 & 1384 & $1.20(1.07,1.35)$ & 0.002 & 0.274 & 883 & $1.24(1.08,1.41)$ & 0.002 & 0.254 & 510 & $1.14(0.97,1.34)$ & 0.106 & 0.824 \\
\hline & Dominant & 1393 & 1384 & $1.22(1.05,1.43)$ & 0.009 & 0.561 & 883 & $1.27(1.07,1.50)$ & 0.007 & 0.571 & 510 & $1.16(0.94,1.43)$ & 0.169 & 0.896 \\
\hline & Recessive & 1393 & 1384 & $1.38(1.05,1.80)$ & 0.020 & 0.898 & 883 & $1.46(1.08,1.97)$ & 0.013 & 0.827 & 510 & $1.27(0.88,1.85)$ & 0.204 & 0.939 \\
\hline \multirow[t]{6}{*}{ rs1801224 } & AA & 601 & 669 & 1.00 (ref) & 0.015 & 0.677 & 359 & 1.00 (ref) & 0.004 & 0.473 & 242 & 1.00 (ref) & 0.517 & 0.998 \\
\hline & $\mathrm{AC}$ & 614 & 582 & $1.18(1.00,1.38)$ & & & 399 & $1.26(1.05,1.51)$ & & & 215 & $1.04(0.84,1.30)$ & & \\
\hline & CC & 180 & 144 & $1.40(1.09,1.80)$ & & & 120 & $1.52(1.15,2.01)$ & & & 60 & $1.22(0.87,1.73)$ & & \\
\hline & Additive & 1395 & 1395 & $1.18(1.06,1.32)$ & 0.004 & 0.374 & 878 & $1.24(1.09,1.41)$ & 0.001 & 0.169 & 517 & $1.08(0.93,1.27)$ & 0.308 & 0.926 \\
\hline & Dominant & 1395 & 1395 & $1.22(1.05,1.42)$ & 0.010 & 0.561 & 878 & $1.31(1.10,1.56)$ & 0.002 & 0.377 & 517 & $1.07(0.87,1.32)$ & 0.494 & 0.965 \\
\hline & Recessive & 1395 & 1395 & $1.29(1.02,1.63)$ & 0.035 & 0.898 & 878 & $1.35(1.04,1.76)$ & 0.026 & 0.875 & 517 & $1.20(0.86,1.67)$ & 0.276 & 0.986 \\
\hline \multirow[t]{6}{*}{ rs7096079 } & CC & 275 & 338 & 1.00 (ref) & 0.023 & 0.677 & 159 & 1.00 (ref) & 0.007 & 0.509 & 116 & 1.00 (ref) & 0.587 & 0.998 \\
\hline & CA & 654 & 620 & $1.31(1.08,1.60)$ & & & 417 & $1.43(1.14,1.80)$ & & & 237 & $1.13(0.87,1.48)$ & & \\
\hline & AA & 301 & 305 & $1.22(0.97,1.53)$ & & & 194 & $1.36(1.04,1.77)$ & & & 107 & $1.03(0.75,1.40)$ & & \\
\hline & Additive & 1230 & 1263 & $1.10(0.99,1.24)$ & 0.084 & 0.894 & 770 & $1.16(1.02,1.32)$ & 0.025 & 0.712 & 460 & $1.02(0.87,1.18)$ & 0.841 & 0.996 \\
\hline & Dominant & 1230 & 1263 & $1.28(1.07,1.54)$ & 0.008 & 0.561 & 770 & $1.41(1.13,1.75)$ & 0.002 & 0.377 & 460 & $1.10(0.86,1.41)$ & 0.461 & 0.965 \\
\hline & Recessive & 1230 & 1263 & $1.02(0.85,1.22)$ & 0.861 & 1.000 & 770 & $1.06(0.86,1.31)$ & 0.578 & 0.991 & 460 & $0.95(0.73,1.22)$ & 0.668 & 0.997 \\
\hline \multicolumn{15}{|l|}{$V D R$} \\
\hline \multirow[t]{6}{*}{ rs 886441} & AA & 885 & 926 & 1.00 (ref) & 0.024 & 0.677 & 563 & 1.00 (ref) & 0.028 & 0.729 & 322 & 1.00 (ref) & 0.179 & 0.963 \\
\hline & AG & 444 & 404 & $1.16(0.98,1.36)$ & & & 273 & $1.12(0.93,1.35)$ & & & 171 & $1.21(0.97,1.52)$ & & \\
\hline & GG & 57 & 36 & $1.66(1.08,2.56)$ & & & 40 & $1.83(1.15,2.93)$ & & & 17 & $1.32(0.73,2.41)$ & & \\
\hline & Additive & 1386 & 1366 & $1.20(1.05,1.38)$ & 0.009 & 0.508 & 876 & $1.20(1.03,1.40)$ & 0.020 & 0.670 & 510 & $1.19(0.99,1.44)$ & 0.067 & 0.690 \\
\hline & Dominant & 1386 & 1366 & $1.20(1.02,1.40)$ & 0.027 & 0.693 & 876 & $1.18(0.98,1.41)$ & 0.078 & 0.896 & 510 & $1.22(0.99,1.52)$ & 0.067 & 0.750 \\
\hline & Recessive & 1386 & 1366 & $1.59(1.04,2.43)$ & 0.034 & 0.898 & 876 & $1.77(1.11,2.81)$ & 0.016 & 0.827 & 510 & $1.24(0.68,2.25)$ & 0.481 & 0.997 \\
\hline \multirow{6}{*}{$\begin{array}{c}\text { NCOA2 } \\
\text { rs10087049 }\end{array}$} & & & & & & & & & & & & & & \\
\hline & AA & 393 & 472 & 1.00 (ref) & 0.007 & 0.569 & 240 & 1.00 (ref) & 0.003 & 0.448 & 153 & 1.00 (ref) & 0.180 & 0.963 \\
\hline & AG & 724 & 665 & $1.32(1.11,1.56)$ & & & 464 & $1.40(1.15,1.71)$ & & & 260 & $1.17(0.92,1.48)$ & & \\
\hline & GG & 173 & 182 & $1.14(0.89,1.46)$ & & & 120 & $1.30(0.98,1.72)$ & & & 53 & $0.88(0.61,1.26)$ & & \\
\hline & Additive & 1290 & 1319 & $1.12(1.00,1.26)$ & 0.054 & 0.848 & 824 & $1.19(1.04,1.36)$ & 0.010 & 0.596 & 466 & $1.00(0.85,1.17)$ & 0.959 & 0.996 \\
\hline & Dominant & 1290 & 1319 & $1.28(1.08,1.51)$ & 0.004 & 0.469 & 824 & $1.38(1.14,1.67)$ & 0.001 & 0.297 & 466 & $1.11(0.88,1.39)$ & 0.385 & 0.963 \\
\hline
\end{tabular}


Table 3. Cont.

\begin{tabular}{|c|c|c|c|c|c|c|c|c|c|c|c|c|c|c|}
\hline \multirow{2}{*}{ Gene/SNP } & \multirow{2}{*}{ Genotype } & \multicolumn{5}{|c|}{ Colorectal Cancer } & \multicolumn{4}{|c|}{ Colon Cancer } & \multicolumn{4}{|c|}{ Rectal Cancer } \\
\hline & & Cases & Controls & OR $(95 \% \mathrm{CI})^{\mathrm{a}}$ & $P$ & $P_{B H}$ & Cases & OR $(95 \% \mathrm{CI})^{\mathrm{a}}$ & $P$ & $P_{B H}$ & Cases & OR $(95 \% \text { CI })^{a}$ & $P$ & $P_{B H}^{\mathrm{b}}$ \\
\hline \multicolumn{15}{|l|}{ NCOA7 } \\
\hline \multirow[t]{6}{*}{ rs10223441 } & $\mathrm{CC}$ & 648 & 709 & 1.00 (ref) & 0.007 & 0.569 & 399 & 1.00 (ref) & 0.009 & 0.531 & 249 & 1.00 (ref) & 0.153 & 0.939 \\
\hline & CG & 640 & 561 & $1.25(1.07,1.47)$ & & & 413 & $1.30(1.09,1.55)$ & & & 227 & $1.17(0.95,1.45)$ & & \\
\hline & GG & 128 & 149 & $0.93(0.72,1.21)$ & & & 84 & $0.98(0.72,1.31)$ & & & 44 & $0.85(0.59,1.23)$ & & \\
\hline & Additive & 1416 & 1419 & $1.06(0.95,1.19)$ & 0.277 & 0.921 & 896 & $1.09(0.96,1.24)$ & 0.183 & 0.920 & 520 & $1.01(0.87,1.18)$ & 0.882 & 0.996 \\
\hline & Dominant & 1416 & 1419 & $1.19(1.02,1.38)$ & 0.025 & 0.669 & 896 & $1.23(1.04,1.46)$ & 0.017 & 0.685 & 520 & $1.10(0.90,1.35)$ & 0.338 & 0.944 \\
\hline & Recessive & 1416 & 1419 & $0.84(0.65,1.08)$ & 0.169 & 0.948 & 896 & $0.86(0.65,1.14)$ & 0.295 & 0.991 & 520 & $0.79(0.55,1.13)$ & 0.202 & 0.939 \\
\hline \multirow{6}{*}{ rs17292488 } & GG & 594 & 639 & 1.00 (ref) & 0.004 & 0.569 & 375 & 1.00 (ref) & 0.019 & 0.659 & 219 & 1.00 (ref) & 0.031 & 0.882 \\
\hline & GA & 657 & 575 & $1.23(1.05,1.44)$ & & & 416 & $1.21(1.01,1.46)$ & & & 241 & $1.26(1.01,1.57)$ & & \\
\hline & AA & 148 & 185 & $0.86(0.67,1.10)$ & & & 97 & $0.86(0.65,1.13)$ & & & 51 & $0.85(0.60,1.21)$ & & \\
\hline & Additive & 1399 & 1399 & $1.01(0.90,1.13)$ & 0.841 & 0.998 & 888 & $1.00(0.89,1.14)$ & 0.942 & 0.994 & 511 & $1.02(0.88,1.19)$ & 0.802 & 0.996 \\
\hline & Dominant & 1399 & 1399 & $1.14(0.98,1.33)$ & 0.087 & 0.876 & 888 & $1.13(0.95,1.34)$ & 0.174 & 0.944 & 511 & $1.16(0.94,1.43)$ & 0.155 & 0.896 \\
\hline & Recessive & 1399 & 1399 & $0.77(0.61,0.97)$ & 0.028 & 0.898 & 888 & $0.77(0.59,1.01)$ & 0.059 & 0.973 & 511 & $0.76(0.54,1.06)$ & 0.102 & 0.939 \\
\hline \multirow{7}{*}{$\begin{array}{c}\text { NCOR2 } \\
\text { rs10846670 }\end{array}$} & & & & & & & & & & & & & & \\
\hline & AA & 288 & 360 & 1.00 (ref) & 0.021 & 0.677 & 169 & 1.00 (ref) & 0.007 & 0.509 & 119 & 1.00 (ref) & 0.676 & 0.998 \\
\hline & AG & 688 & 669 & $1.29(1.07,1.56)$ & & & 441 & $1.39(1.12,1.74)$ & & & 247 & $1.12(0.86,1.44)$ & & \\
\hline & GG & 274 & 265 & $1.30(1.03,1.64)$ & & & 177 & $1.42(1.09,1.86)$ & & & 97 & $1.12(0.81,1.53)$ & & \\
\hline & Additive & 1250 & 1294 & $1.15(1.02,1.29)$ & 0.020 & 0.663 & 787 & $1.20(1.05,1.37)$ & 0.007 & 0.564 & 463 & $1.06(0.91,1.24)$ & 0.461 & 0.927 \\
\hline & Dominant & 1250 & 1294 & $1.29(1.08,1.55)$ & 0.005 & 0.528 & 787 & $1.40(1.13,1.73)$ & 0.002 & 0.377 & 463 & $1.12(0.87,1.43)$ & 0.377 & 0.962 \\
\hline & Recessive & 1250 & 1294 & $1.09(0.90,1.32)$ & 0.356 & 0.987 & 787 & $1.13(0.91,1.41)$ & 0.256 & 0.991 & 463 & $1.04(0.80,1.36)$ & 0.777 & 0.997 \\
\hline \multirow[t]{6}{*}{ rs906304 } & GG & 1032 & 1082 & 1.00 (ref) & 0.010 & 0.569 & 666 & 1.00 (ref) & 0.005 & 0.496 & 366 & 1.00 (ref) & 0.025 & 0.827 \\
\hline & GA & 359 & 298 & $1.26(1.06,1.51)$ & & & 220 & $1.20(0.98,1.47)$ & & & 139 & $1.37(1.08,1.74)$ & & \\
\hline & AA & 20 & 32 & $0.66(0.38,1.17)$ & & & 6 & $0.30(0.13,0.74)$ & & & 14 & $1.36(0.71,2.62)$ & & \\
\hline & Additive & 1411 & 1412 & $1.12(0.96,1.31)$ & 0.141 & 0.894 & 892 & $1.02(0.85,1.22)$ & 0.822 & 0.990 & 519 & $1.30(1.07,1.59)$ & 0.010 & 0.514 \\
\hline & Dominant & 1411 & 1412 & $1.21(1.02,1.43)$ & 0.032 & 0.693 & 892 & $1.12(0.92,1.36)$ & 0.268 & 0.954 & 519 & $1.37(1.09,1.73)$ & 0.007 & 0.416 \\
\hline & Recessive & 1411 & 1412 & $0.63(0.35,1.11)$ & 0.106 & 0.931 & 892 & $0.29(0.12,0.70)$ & 0.006 & 0.714 & 519 & $1.26(0.66,2.40)$ & 0.490 & 0.997 \\
\hline \multicolumn{15}{|l|}{ CHAF1A } \\
\hline \multirow[t]{6}{*}{ rs243352 } & $\mathrm{CC}$ & 410 & 369 & 1.00 (ref) & 0.014 & 0.677 & 250 & 1.00 (ref) & 0.240 & 0.977 & 160 & 1.00 (ref) & 0.003 & 0.453 \\
\hline & $\mathrm{CA}$ & 695 & 673 & $0.93(0.78,1.11)$ & & & 438 & $0.97(0.79,1.19)$ & & & 257 & $0.85(0.67,1.08)$ & & \\
\hline & AA & 285 & 346 & $0.74(0.60,0.91)$ & & & 190 & $0.82(0.65,1.05)$ & & & 95 & $0.60(0.44,0.80)$ & & \\
\hline & Additive & 1390 & 1388 & $0.86(0.78,0.96)$ & 0.006 & 0.417 & 878 & $0.91(0.81,1.03)$ & 0.128 & 0.920 & 512 & $0.78(0.67,0.90)$ & 0.001 & 0.132 \\
\hline & Dominant & 1390 & 1388 & $0.86(0.73,1.02)$ & 0.087 & 0.876 & 878 & $0.92(0.76,1.11)$ & 0.392 & 0.956 & 512 & $0.76(0.61,0.95)$ & 0.018 & 0.629 \\
\hline & Recessive & 1390 & 1388 & $0.77(0.65,0.92)$ & 0.005 & 0.648 & 878 & $0.84(0.69,1.03)$ & 0.097 & 0.981 & 512 & $0.66(0.51,0.86)$ & 0.002 & 0.729 \\
\hline \multirow[t]{6}{*}{ rs9352 } & AA & 461 & 417 & 1.00 (ref) & 0.023 & 0.677 & 277 & 1.00 (ref) & 0.315 & 0.995 & 184 & 1.00 (ref) & 0.003 & 0.453 \\
\hline & $\mathrm{AG}$ & 648 & 681 & $0.86(0.72,1.02)$ & & & 413 & $0.92(0.76,1.13)$ & & & 235 & $0.75(0.60,0.95)$ & & \\
\hline & GG & 254 & 307 & $0.74(0.60,0.92)$ & & & 166 & $0.83(0.65,1.06)$ & & & 88 & $0.61(0.46,0.83)$ & & \\
\hline & Additive & 1363 & 1405 & $0.86(0.78,0.96)$ & 0.006 & 0.417 & 856 & $0.91(0.81,1.03)$ & 0.131 & 0.920 & 507 & $0.78(0.67,0.90)$ & 0.001 & 0.132 \\
\hline & Dominant & 1363 & 1405 & $0.82(0.70,0.97)$ & 0.018 & 0.597 & 856 & $0.89(0.74,1.08)$ & 0.238 & 0.954 & 507 & $0.71(0.57,0.88)$ & 0.002 & 0.243 \\
\hline & Recessive & 1363 & 1405 & $0.81(0.68,0.98)$ & 0.032 & 0.898 & 856 & $0.87(0.70,1.07)$ & 0.192 & 0.991 & 507 & $0.73(0.56,0.95)$ & 0.018 & 0.939 \\
\hline
\end{tabular}


Table 3. Cont

\begin{tabular}{|c|c|c|c|c|c|c|c|c|c|c|c|c|c|c|}
\hline \multirow{2}{*}{ Gene/SNP } & \multirow{2}{*}{ Genotype } & \multicolumn{5}{|c|}{ Colorectal Cancer } & \multicolumn{4}{|c|}{ Colon Cancer } & \multicolumn{4}{|c|}{ Rectal Cancer } \\
\hline & & Cases & Controls & OR $(95 \% \mathrm{CI})^{\mathrm{a}}$ & $P$ & $P_{B H}$ & Cases & OR $(95 \% \mathrm{CI})^{\mathrm{a}}$ & $P$ & $P_{B H}$ & Cases & OR $(95 \% C I)^{a}$ & $P$ & $P_{B H}{ }^{b}$ \\
\hline \multicolumn{15}{|l|}{ HDAC9 } \\
\hline \multirow[t]{6}{*}{ rs2520361 } & AA & 881 & 841 & 1.00 (ref) & 0.021 & 0.677 & 556 & 1.00 (ref) & 0.161 & 0.960 & 325 & 1.00 (ref) & 0.033 & 0.882 \\
\hline & AG & 385 & 395 & $0.92(0.78,1.09)$ & & & 240 & $0.93(0.76,1.13)$ & & & 145 & $0.91(0.72,1.15)$ & & \\
\hline & GG & 50 & 79 & $0.60(0.41,0.87)$ & & & 36 & $0.68(0.45,1.02)$ & & & 14 & $0.46(0.26,0.83)$ & & \\
\hline & Additive & 1316 & 1315 & $0.85(0.75,0.97)$ & 0.018 & 0.641 & 832 & $0.88(0.75,1.02)$ & 0.086 & 0.920 & 484 & $0.81(0.67,0.98)$ & 0.027 & 0.664 \\
\hline & Dominant & 1316 & 1315 & $0.87(0.74,1.02)$ & 0.089 & 0.876 & 832 & $0.89(0.74,1.07)$ & 0.197 & 0.946 & 484 & $0.84(0.67,1.05)$ & 0.122 & 0.853 \\
\hline & Recessive & 1316 & 1315 & $0.61(0.43,0.88)$ & 0.009 & 0.898 & 832 & $0.69(0.46,1.04)$ & 0.079 & 0.973 & 484 & $0.48(0.27,0.85)$ & 0.013 & 0.939 \\
\hline \multirow{6}{*}{ rs4141042 } & AA & 1028 & 1072 & 1.00 (ref) & 0.007 & 0.569 & 645 & 1.00 (ref) & 0.006 & 0.509 & 383 & 1.00 (ref) & 0.146 & 0.921 \\
\hline & AG & 366 & 304 & $1.26(1.06,1.50)$ & & & 238 & $1.30(1.07,1.58)$ & & & 128 & $1.23(0.97,1.57)$ & & \\
\hline & GG & 18 & 31 & $0.60(0.33,1.09)$ & & & 10 & $0.54(0.26,1.12)$ & & & 8 & $0.71(0.32,1.56)$ & & \\
\hline & Additive & 1412 & 1407 & $1.11(0.95,1.30)$ & 0.176 & 0.916 & 893 & $1.13(0.95,1.35)$ & 0.166 & 0.920 & 519 & $1.10(0.90,1.36)$ & 0.346 & 0.926 \\
\hline & Dominant & 1412 & 1407 & $1.20(1.01,1.42)$ & 0.039 & 0.752 & 893 & $1.23(1.01,1.49)$ & 0.035 & 0.806 & 519 & $1.18(0.93,1.49)$ & 0.164 & 0.896 \\
\hline & Recessive & 1412 & 1407 & $0.57(0.32,1.03)$ & 0.062 & 0.916 & 893 & $0.51(0.25,1.05)$ & 0.067 & 0.973 & 519 & $0.67(0.30,1.49)$ & 0.328 & 0.986 \\
\hline \multicolumn{15}{|l|}{ SMARCC1 } \\
\hline \multirow[t]{6}{*}{ rs3755637 } & GG & 661 & 605 & 1.00 (ref) & 0.015 & 0.677 & 412 & 1.00 (ref) & 0.026 & 0.729 & 249 & 1.00 (ref) & 0.073 & 0.882 \\
\hline & GA & 520 & 601 & $0.79(0.67,0.93)$ & & & 322 & $0.78(0.64,0.93)$ & & & 198 & $0.81(0.65,1.02)$ & & \\
\hline & AA & 132 & 141 & $0.86(0.66,1.12)$ & & & 90 & $0.95(0.70,1.27)$ & & & 42 & $0.70(0.48,1.03)$ & & \\
\hline & Additive & 1313 & 1347 & $0.87(0.78,0.98)$ & 0.023 & 0.712 & 824 & $0.90(0.79,1.03)$ & 0.111 & 0.920 & 489 & $0.83(0.70,0.97)$ & 0.023 & 0.650 \\
\hline & Dominant & 1313 & 1347 & $0.80(0.69,0.93)$ & 0.005 & 0.517 & 824 & $0.81(0.68,0.96)$ & 0.017 & 0.685 & 489 & $0.79(0.64,0.98)$ & 0.030 & 0.679 \\
\hline & Recessive & 1313 & 1347 & $0.96(0.75,1.24)$ & 0.758 & 1.000 & 824 & $1.07(0.80,1.41)$ & 0.659 & 0.991 & 489 & $0.78(0.54,1.12)$ & 0.174 & 0.939 \\
\hline \multirow{7}{*}{$\begin{array}{c}\text { TOP2B } \\
\text { rs1001647 }\end{array}$} & & & & & & & & & & & & & & \\
\hline & AA & 948 & 884 & 1.00 (ref) & 0.022 & 0.677 & 612 & 1.00 (ref) & 0.011 & 0.531 & 336 & 1.00 (ref) & 0.460 & 0.993 \\
\hline & AG & 353 & 415 & $0.79(0.66,0.93)$ & & & 220 & $0.74(0.61,0.90)$ & & & 133 & $0.87(0.68,1.10)$ & & \\
\hline & GG & 57 & 55 & $0.94(0.64,1.39)$ & & & 37 & $0.91(0.59,1.41)$ & & & 20 & $1.06(0.61,1.81)$ & & \\
\hline & Additive & 1358 & 1354 & $0.86(0.75,0.99)$ & 0.032 & 0.792 & 869 & $0.83(0.71,0.97)$ & 0.017 & 0.650 & 489 & $0.93(0.77,1.13)$ & 0.454 & 0.926 \\
\hline & Dominant & 1358 & 1354 & $0.80(0.68,0.95)$ & 0.009 & 0.561 & 869 & $0.76(0.63,0.92)$ & 0.004 & 0.442 & 489 & $0.89(0.71,1.11)$ & 0.298 & 0.943 \\
\hline & Recessive & 1358 & 1354 & $1.02(0.70,1.49)$ & 0.922 & 1.000 & 869 & $1.00(0.65,1.55)$ & 0.982 & 0.999 & 489 & $1.11(0.65,1.90)$ & 0.702 & 0.997 \\
\hline
\end{tabular}

${ }^{a}$ Unconditional logistic regression adjusted for age at blood collection, sex, and study center. ${ }^{b} P$ of false discovery rate (BH; Benjamini-Hochberg) method. 
Table 4. P-values of pathway- and gene-level associations with CRC risk overall and by tumor location (colon vs. rectal) and of interactions with $25(\mathrm{OH}) \mathrm{D}$ concentrations (per $24.96 \mathrm{nmol} / \mathrm{L}$ ), the EPIC study, 1992-2003.

\begin{tabular}{|c|c|c|c|c|c|c|c|c|c|c|c|c|c|c|}
\hline \multirow{3}{*}{ Pathway/Gene } & \multirow{3}{*}{$\begin{array}{l}\text { No. of } \\
\text { SNPs }\end{array}$} & \multirow{3}{*}{$\begin{array}{l}\text { No. of SNPs } \\
\text { Retained After } \\
\text { Pruning }\end{array}$} & \multicolumn{4}{|c|}{ Colorectal Cancer } & \multicolumn{4}{|c|}{ Colon Cancer } & \multicolumn{4}{|c|}{ Rectal Cancer } \\
\hline & & & \multicolumn{2}{|c|}{$\begin{array}{c}\text { Gene or } \\
\text { Pathway Only }\end{array}$} & \multicolumn{2}{|c|}{$\begin{array}{c}\text { Gene- or } \\
\text { Pathway-25(OH)D } \\
\text { Interaction }\end{array}$} & \multicolumn{2}{|c|}{$\begin{array}{c}\text { Gene or } \\
\text { Pathway Only }\end{array}$} & \multicolumn{2}{|c|}{$\begin{array}{c}\text { Gene- or } \\
\text { Pathway-25(OH)D } \\
\text { Interaction }\end{array}$} & \multicolumn{2}{|c|}{$\begin{array}{c}\text { Gene or } \\
\text { Pathway Only }\end{array}$} & \multicolumn{2}{|c|}{$\begin{array}{c}\text { Gene- or } \\
\text { Pathway-25(OH)D } \\
\text { Interaction }\end{array}$} \\
\hline & & & $P$ & $P_{B H}{ }^{a}$ & $P$ & $P_{B H}$ & $P$ & $P_{B H}$ & $P$ & $P_{B H}$ & $P$ & $P_{B H}$ & $P$ & $P_{B H}$ \\
\hline Vitamin D metabolism & 276 & 245 & 0.580 & & 0.159 & & 0.550 & & 0.160 & & 0.418 & & 0.116 & \\
\hline Identified in GWAS of $25(\mathrm{OH}) \mathrm{D}$ & 2 & 2 & 0.235 & 0.759 & 0.867 & 0.999 & 0.167 & 0.657 & 0.923 & 0.990 & 0.561 & 0.944 & 0.499 & 0.991 \\
\hline CUBN & 116 & 106 & 0.173 & 0.741 & 0.764 & 0.999 & 0.130 & 0.657 & 0.896 & 0.990 & 0.083 & 0.470 & 0.490 & 0.991 \\
\hline CYP24A1 & 25 & 23 & 0.443 & 0.777 & 0.358 & 0.999 & 0.083 & 0.647 & 0.666 & 0.990 & 0.622 & 0.944 & 0.007 & 0.595 \\
\hline CYP27A1 & 5 & 5 & 0.500 & 0.777 & 0.299 & 0.999 & 0.488 & 0.819 & 0.086 & 0.522 & 0.968 & 0.968 & 0.256 & 0.991 \\
\hline CYP27B1 & 6 & 5 & 0.448 & 0.777 & 0.037 & 0.446 & 0.585 & 0.829 & 0.041 & 0.448 & 0.514 & 0.944 & 0.154 & 0.991 \\
\hline CYP2R1 & 12 & 9 & 0.115 & 0.741 & 0.811 & 0.999 & 0.368 & 0.815 & 0.921 & 0.990 & 0.044 & 0.459 & 0.727 & 0.991 \\
\hline CYP3A4 & 7 & 5 & 0.241 & 0.759 & 0.730 & 0.999 & 0.461 & 0.815 & 0.533 & 0.990 & 0.262 & 0.747 & 0.392 & 0.991 \\
\hline DHCR7 & 12 & 6 & 0.997 & 0.997 & 0.549 & 0.999 & 0.800 & 0.911 & 0.614 & 0.990 & 0.434 & 0.944 & 0.716 & 0.991 \\
\hline GC & 24 & 20 & 0.484 & 0.777 & 0.018 & 0.406 & 0.912 & 0.954 & 0.026 & 0.442 & 0.241 & 0.747 & 0.316 & 0.991 \\
\hline LRP2 & 67 & 64 & 0.804 & 0.926 & 0.377 & 0.999 & 0.508 & 0.819 & 0.677 & 0.990 & 0.904 & 0.967 & 0.487 & 0.991 \\
\hline Mineral homeostasis & 58 & 40 & 0.834 & & 0.313 & & 0.912 & & 0.431 & & 0.537 & & 0.782 & \\
\hline CASR & 31 & 23 & 0.580 & 0.784 & 0.736 & 0.999 & 0.536 & 0.819 & 0.957 & 0.990 & 0.643 & 0.944 & 0.565 & 0.991 \\
\hline PTH & 6 & 5 & 0.931 & 0.982 & 0.671 & 0.999 & 0.773 & 0.911 & 0.739 & 0.990 & 0.539 & 0.944 & 0.736 & 0.991 \\
\hline CALB1 & 2 & 2 & 0.489 & 0.777 & 0.741 & 0.999 & 0.400 & 0.815 & 0.847 & 0.990 & 0.882 & 0.967 & 0.819 & 0.991 \\
\hline TRPV5 & 9 & 7 & 0.657 & 0.846 & 0.054 & 0.456 & 0.920 & 0.954 & 0.081 & 0.522 & 0.337 & 0.818 & 0.225 & 0.991 \\
\hline TRPV6 & 10 & 3 & 0.263 & 0.777 & 0.880 & 0.999 & 0.520 & 0.819 & 0.954 & 0.990 & 0.112 & 0.595 & 0.713 & 0.991 \\
\hline $\begin{array}{l}\text { VDR complex/Transcriptional } \\
\text { Co-regulators and } \\
\text { Co-activators }\end{array}$ & 538 & 490 & 0.634 & & 0.041 & & 0.874 & & 0.105 & & 0.180 & & 0.727 & \\
\hline ACTL6A & 3 & 3 & 0.239 & 0.759 & 0.395 & 0.999 & 0.262 & 0.815 & 0.497 & 0.990 & 0.506 & 0.944 & 0.613 & 0.991 \\
\hline ARID1A & 8 & 7 & 0.408 & 0.777 & 0.032 & 0.446 & 0.306 & 0.815 & 0.048 & 0.448 & 0.133 & 0.628 & 0.068 & 0.924 \\
\hline BAZ1B & 14 & 9 & 0.478 & 0.777 & 0.955 & 0.999 & 0.360 & 0.815 & 0.867 & 0.990 & 0.385 & 0.909 & 0.935 & 0.993 \\
\hline CARM1 & 4 & 4 & 0.641 & 0.839 & 0.006 & 0.406 & 0.831 & 0.929 & 0.022 & 0.442 & 0.290 & 0.747 & 0.120 & 0.991 \\
\hline CHAF1A & 5 & 4 & 0.035 & 0.511 & 0.013 & 0.406 & 0.307 & 0.815 & 0.047 & 0.448 & 0.007 & 0.187 & 0.098 & 0.926 \\
\hline CREBBP & 15 & 12 & 0.388 & 0.777 & 0.285 & 0.999 & 0.800 & 0.911 & 0.215 & 0.865 & 0.011 & 0.187 & 0.793 & 0.991 \\
\hline EP300 & 6 & 5 & 0.771 & 0.926 & 0.791 & 0.999 & 0.434 & 0.815 & 0.835 & 0.990 & 0.917 & 0.967 & 0.342 & 0.991 \\
\hline HDAC9 & 149 & 141 & 0.559 & 0.784 & 0.873 & 0.999 & 0.524 & 0.819 & 0.578 & 0.990 & 0.553 & 0.944 & 0.970 & 0.993 \\
\hline
\end{tabular}


Table 4. Cont

\begin{tabular}{|c|c|c|c|c|c|c|c|c|c|c|c|c|c|c|}
\hline \multirow{3}{*}{ Pathway/Gene } & \multirow{3}{*}{$\begin{array}{l}\text { No. of } \\
\text { SNPs }\end{array}$} & \multirow{3}{*}{$\begin{array}{l}\text { No. of SNPs } \\
\text { Retained After } \\
\text { Pruning }\end{array}$} & \multicolumn{4}{|c|}{ Colorectal Cancer } & \multicolumn{4}{|c|}{ Colon Cancer } & \multicolumn{4}{|c|}{ Rectal Cancer } \\
\hline & & & \multicolumn{2}{|c|}{$\begin{array}{c}\text { Gene or } \\
\text { Pathway Only }\end{array}$} & \multicolumn{2}{|c|}{$\begin{array}{c}\text { Gene- or } \\
\text { Pathway-25(OH)D } \\
\text { Interaction }\end{array}$} & \multicolumn{2}{|c|}{$\begin{array}{c}\text { Gene or } \\
\text { Pathway Only }\end{array}$} & \multicolumn{2}{|c|}{$\begin{array}{c}\text { Gene- or } \\
\text { Pathway-25(OH)D } \\
\text { Interaction }\end{array}$} & \multicolumn{2}{|c|}{$\begin{array}{c}\text { Gene or } \\
\text { Pathway Only }\end{array}$} & \multicolumn{2}{|c|}{$\begin{array}{c}\text { Gene- or } \\
\text { Pathway-25(OH)D } \\
\text { Interaction }\end{array}$} \\
\hline & & & $P$ & $P_{B H}{ }^{a}$ & $P$ & $P_{B H}$ & $P$ & $P_{B H}$ & $P$ & $P_{B H}$ & $P$ & $P_{B H}$ & $P$ & $P_{B H}$ \\
\hline MED1 & 5 & 5 & 0.507 & 0.777 & 0.561 & 0.999 & 0.719 & 0.899 & 0.694 & 0.990 & 0.131 & 0.628 & 0.617 & 0.991 \\
\hline NCOA1 & 18 & 14 & 0.581 & 0.784 & 0.065 & 0.504 & 0.804 & 0.911 & 0.201 & 0.854 & 0.239 & 0.747 & 0.355 & 0.991 \\
\hline $\mathrm{NCOA} 2$ & 19 & 16 & 0.542 & 0.781 & 0.579 & 0.999 & 0.145 & 0.657 & 0.456 & 0.990 & 0.938 & 0.967 & 0.505 & 0.991 \\
\hline NCOA3 & 11 & 9 & 0.067 & 0.636 & 0.051 & 0.456 & 0.056 & 0.647 & 0.069 & 0.489 & 0.522 & 0.944 & 0.350 & 0.991 \\
\hline NCOA7 & 31 & 31 & 0.518 & 0.777 & 0.142 & 0.755 & 0.646 & 0.872 & 0.056 & 0.448 & 0.223 & 0.747 & 0.482 & 0.991 \\
\hline NCOR1 & 7 & 3 & 0.312 & 0.777 & 0.802 & 0.999 & 0.377 & 0.815 & 0.759 & 0.990 & 0.577 & 0.944 & 0.489 & 0.991 \\
\hline PCAF/KAT2B & 31 & 31 & 0.801 & 0.926 & 0.891 & 0.999 & 0.470 & 0.815 & 0.865 & 0.990 & 0.938 & 0.967 & 0.797 & 0.991 \\
\hline PRMT1 & 4 & 4 & 0.346 & 0.777 & 0.351 & 0.999 & 0.407 & 0.815 & 0.184 & 0.823 & 0.618 & 0.944 & 0.624 & 0.991 \\
\hline RXRA & 30 & 27 & 0.683 & 0.866 & 0.763 & 0.999 & 0.091 & 0.647 & 0.732 & 0.990 & 0.662 & 0.944 & 0.726 & 0.991 \\
\hline RXRB & 7 & 3 & 0.824 & 0.926 & 0.074 & 0.526 & 0.445 & 0.815 & 0.058 & 0.448 & 0.617 & 0.944 & 0.356 & 0.991 \\
\hline RXRG & 24 & 24 & 0.853 & 0.929 & 0.716 & 0.999 & 0.558 & 0.819 & 0.923 & 0.990 & 0.875 & 0.967 & 0.087 & 0.924 \\
\hline SMARCA2 & 1 & 1 & 0.506 & 0.777 & 0.019 & 0.406 & 0.381 & 0.815 & 0.012 & 0.442 & 0.944 & 0.967 & 0.307 & 0.991 \\
\hline SMARCA4 & 12 & 9 & 0.474 & 0.777 & 0.794 & 0.999 & 0.559 & 0.819 & 0.975 & 0.990 & 0.557 & 0.944 & 0.923 & 0.993 \\
\hline SMARCC1 & 4 & 4 & 0.103 & 0.741 & 0.893 & 0.999 & 0.344 & 0.815 & 0.824 & 0.990 & 0.082 & 0.470 & 0.164 & 0.991 \\
\hline SMARCD1 & 3 & 3 & 0.312 & 0.777 & 0.703 & 0.999 & 0.356 & 0.815 & 0.550 & 0.990 & 0.519 & 0.944 & 0.998 & 0.998 \\
\hline SMARCE1 & 4 & 4 & 0.048 & 0.582 & 0.197 & 0.881 & 0.191 & 0.706 & 0.390 & 0.990 & 0.083 & 0.470 & 0.059 & 0.924 \\
\hline SNW1 & 10 & 10 & 0.615 & 0.816 & 0.863 & 0.999 & 0.740 & 0.911 & 0.947 & 0.990 & 0.813 & 0.967 & 0.807 & 0.991 \\
\hline SUPT16H & 7 & 6 & 0.809 & 0.926 & 0.990 & 0.999 & 0.696 & 0.899 & 0.960 & 0.990 & 0.663 & 0.944 & 0.841 & 0.991 \\
\hline TOP2B & 6 & 5 & 0.192 & 0.741 & 0.965 & 0.999 & 0.097 & 0.647 & 0.327 & 0.990 & 0.281 & 0.747 & 0.400 & 0.991 \\
\hline NCOR2 & 62 & 61 & 0.701 & 0.877 & 0.960 & 0.999 & 0.770 & 0.911 & 0.609 & 0.990 & 0.459 & 0.944 & 0.935 & 0.993 \\
\hline VDR & 30 & 28 & 0.270 & 0.777 & 0.652 & 0.999 & 0.372 & 0.815 & 0.707 & 0.990 & 0.477 & 0.944 & 0.706 & 0.991 \\
\hline TSC2 & 8 & 7 & 0.135 & 0.741 & 0.885 & 0.999 & 0.354 & 0.815 & 0.811 & 0.990 & 0.284 & 0.747 & 0.761 & 0.991 \\
\hline TGF-beta signaling & 110 & 98 & 0.0001 & & 0.616 & & 0.0001 & & 0.729 & & 0.0001 & & 0.342 & \\
\hline RHPN2 & 25 & 23 & 0.452 & 0.777 & 0.722 & 0.999 & 0.144 & 0.657 & 0.871 & 0.990 & 0.956 & 0.967 & 0.701 & 0.991 \\
\hline SMAD7 & 23 & 18 & 0.0001 & 0.008 & 0.927 & 0.999 & 0.001 & 0.043 & 0.947 & 0.990 & 0.0005 & 0.021 & 0.627 & 0.991 \\
\hline SMAD3 & 39 & 38 & 0.0002 & 0.008 & 0.367 & 0.999 & 0.0001 & 0.009 & 0.514 & 0.990 & 0.0003 & 0.021 & 0.085 & 0.924 \\
\hline BMP2 & 5 & 5 & 0.016 & 0.346 & 0.081 & 0.527 & 0.014 & 0.298 & 0.224 & 0.865 & 0.072 & 0.470 & 0.049 & 0.924 \\
\hline BMP4 & 1 & 1 & 0.201 & 0.742 & 0.431 & 0.999 & 0.334 & 0.815 & 0.615 & 0.990 & 0.261 & 0.747 & 0.276 & 0.991 \\
\hline TGFB1 & 11 & 8 & 0.159 & 0.741 & 0.293 & 0.999 & 0.469 & 0.815 & 0.112 & 0.595 & 0.068 & 0.470 & 0.306 & 0.991 \\
\hline TGFBR1 & 4 & 3 & 0.950 & 0.982 & 0.741 & 0.999 & 0.306 & 0.815 & 0.838 & 0.990 & 0.446 & 0.944 & 0.851 & 0.991 \\
\hline SCG5/GREM1 & 2 & 2 & 0.141 & 0.741 & 0.412 & 0.999 & 0.170 & 0.657 & 0.371 & 0.990 & 0.323 & 0.808 & 0.656 & 0.991 \\
\hline
\end{tabular}


Table 4. Cont

\begin{tabular}{|c|c|c|c|c|c|c|c|c|c|c|c|c|c|c|}
\hline \multirow{3}{*}{ Pathway/Gene } & \multirow{3}{*}{$\begin{array}{l}\text { No. of } \\
\text { SNPs }\end{array}$} & \multirow{3}{*}{$\begin{array}{l}\text { No. of SNPs } \\
\text { Retained After } \\
\text { Pruning }\end{array}$} & \multicolumn{4}{|c|}{ Colorectal Cancer } & \multicolumn{4}{|c|}{ Colon Cancer } & \multicolumn{4}{|c|}{ Rectal Cancer } \\
\hline & & & \multicolumn{2}{|c|}{$\begin{array}{c}\text { Gene or } \\
\text { Pathway Only }\end{array}$} & \multicolumn{2}{|c|}{$\begin{array}{c}\text { Gene- or } \\
\text { Pathway-25(OH)D } \\
\text { Interaction }\end{array}$} & \multicolumn{2}{|c|}{$\begin{array}{c}\text { Gene or } \\
\text { Pathway Only }\end{array}$} & \multicolumn{2}{|c|}{$\begin{array}{c}\text { Gene- or } \\
\text { Pathway-25(OH)D } \\
\text { Interaction }\end{array}$} & \multicolumn{2}{|c|}{$\begin{array}{c}\text { Gene or } \\
\text { Pathway Only }\end{array}$} & \multicolumn{2}{|c|}{$\begin{array}{c}\text { Gene- or } \\
\text { Pathway-25(OH)D } \\
\text { Interaction }\end{array}$} \\
\hline & & & $P$ & $P_{B H}{ }^{a}$ & $P$ & $P_{B H}$ & $P$ & $P_{B H}$ & $P$ & $P_{B H}$ & $P$ & $P_{B H}$ & $P$ & $P_{B H}$ \\
\hline Inflammation & 133 & 97 & 0.888 & & 0.479 & & 0.620 & & 0.156 & & 0.200 & & 0.784 & \\
\hline ALOX5 & 22 & 11 & 0.839 & 0.926 & 0.264 & 0.999 & 0.801 & 0.911 & 0.593 & 0.990 & 0.782 & 0.967 & 0.165 & 0.991 \\
\hline IL10 & 13 & 8 & 0.462 & 0.777 & 0.030 & 0.446 & 0.854 & 0.931 & 0.006 & 0.442 & 0.009 & 0.187 & 0.419 & 0.991 \\
\hline IL10R & 9 & 7 & 0.734 & 0.904 & 0.552 & 0.999 & 0.913 & 0.954 & 0.751 & 0.990 & 0.663 & 0.944 & 0.591 & 0.991 \\
\hline IL2/IL21 & 6 & 5 & 0.441 & 0.777 & 0.933 & 0.999 & 0.538 & 0.819 & 0.978 & 0.990 & 0.666 & 0.944 & 0.607 & 0.991 \\
\hline IL6 & 13 & 10 & 0.983 & 0.995 & 0.807 & 0.999 & 0.982 & 0.988 & 0.557 & 0.990 & 0.696 & 0.967 & 0.843 & 0.991 \\
\hline IL12B & 18 & 17 & 0.128 & 0.741 & 0.870 & 0.999 & 0.079 & 0.647 & 0.796 & 0.990 & 0.791 & 0.967 & 0.761 & 0.991 \\
\hline IFNG & 7 & 4 & 0.300 & 0.777 & 0.686 & 0.999 & 0.453 & 0.815 & 0.608 & 0.990 & 0.287 & 0.747 & 0.497 & 0.991 \\
\hline TNF & 4 & 4 & 0.959 & 0.982 & 0.218 & 0.926 & 0.988 & 0.988 & 0.375 & 0.990 & 0.790 & 0.967 & 0.076 & 0.924 \\
\hline NFKB1 & 22 & 15 & 0.816 & 0.926 & 0.644 & 0.999 & 0.873 & 0.939 & 0.736 & 0.990 & 0.856 & 0.967 & 0.794 & 0.991 \\
\hline IL12A & 1 & 1 & 0.523 & 0.777 & 0.168 & 0.795 & 0.452 & 0.815 & 0.159 & 0.770 & 0.814 & 0.967 & 0.706 & 0.991 \\
\hline IL18 & 4 & 4 & 0.348 & 0.777 & 0.514 & 0.999 & 0.613 & 0.840 & 0.766 & 0.990 & 0.182 & 0.737 & 0.290 & 0.991 \\
\hline IL1A/IL1B & 11 & 8 & 0.951 & 0.982 & 0.124 & 0.701 & 0.846 & 0.931 & 0.054 & 0.448 & 0.761 & 0.967 & 0.711 & 0.991 \\
\hline IL8 & 1 & 1 & 0.153 & 0.741 & 0.978 & 0.999 & 0.060 & 0.647 & 0.593 & 0.990 & 0.811 & 0.967 & 0.669 & 0.991 \\
\hline RELA (p65) & 2 & 2 & 0.921 & 0.982 & 0.835 & 0.999 & 0.938 & 0.961 & 0.962 & 0.990 & 0.922 & 0.967 & 0.690 & 0.991 \\
\hline Oxidative Stress & 51 & 37 & 0.726 & & 0.471 & & 0.598 & & 0.460 & & 0.913 & & 0.747 & \\
\hline GSR & 9 & 7 & 0.530 & 0.777 & 0.116 & 0.701 & 0.712 & 0.899 & 0.110 & 0.595 & 0.656 & 0.944 & 0.376 & 0.991 \\
\hline GPx2 & 15 & 8 & 0.834 & 0.926 & 0.772 & 0.999 & 0.713 & 0.899 & 0.748 & 0.990 & 0.412 & 0.944 & 0.915 & 0.993 \\
\hline TXNRD1 (TR1) & 17 & 14 & 0.232 & 0.759 & 0.859 & 0.999 & 0.168 & 0.657 & 0.875 & 0.990 & 0.757 & 0.967 & 0.946 & 0.993 \\
\hline SOD2 & 10 & 8 & 0.530 & 0.777 & 0.374 & 0.999 & 0.546 & 0.819 & 0.599 & 0.990 & 0.896 & 0.967 & 0.281 & 0.991 \\
\hline $\begin{array}{l}\text { Insulin growth factor (IGF) } \\
\text { signaling }\end{array}$ & 61 & 52 & 0.105 & & 0.320 & & 0.550 & & 0.135 & & 0.007 & & 0.346 & \\
\hline $\begin{array}{c}\text { Associated with IGF levels in } \\
\text { GWAS }{ }^{c}\end{array}$ & 4 & 4 & 0.414 & 0.777 & 0.999 & 0.999 & 0.579 & 0.829 & 0.901 & 0.990 & 0.036 & 0.437 & 0.879 & 0.993 \\
\hline IGF1 & 17 & 15 & 0.131 & 0.741 & 0.452 & 0.999 & 0.113 & 0.657 & 0.668 & 0.990 & 0.225 & 0.747 & 0.655 & 0.991 \\
\hline IGF2BP2 & 3 & 1 & 0.303 & 0.777 & 0.923 & 0.999 & 0.596 & 0.830 & 0.580 & 0.990 & 0.156 & 0.698 & 0.658 & 0.991 \\
\hline IGFBP2/IGFBP5 & 24 & 21 & 0.187 & 0.741 & 0.052 & 0.456 & 0.692 & 0.899 & 0.018 & 0.442 & 0.029 & 0.411 & 0.607 & 0.991 \\
\hline IGFBP3 & 13 & 11 & 0.057 & 0.601 & 0.882 & 0.999 & 0.273 & 0.815 & 0.997 & 0.997 & 0.050 & 0.459 & 0.058 & 0.924 \\
\hline
\end{tabular}


Table 4. Cont.

\begin{tabular}{|c|c|c|c|c|c|c|c|c|c|c|c|c|c|c|}
\hline \multirow{3}{*}{ Pathway/Gene } & \multirow{3}{*}{$\begin{array}{l}\text { No. of } \\
\text { SNPs }\end{array}$} & \multirow{3}{*}{$\begin{array}{l}\text { No. of SNPs } \\
\text { Retained After } \\
\text { Pruning }\end{array}$} & \multicolumn{4}{|c|}{ Colorectal Cancer } & \multicolumn{4}{|c|}{ Colon Cancer } & \multicolumn{4}{|c|}{ Rectal Cancer } \\
\hline & & & \multicolumn{2}{|c|}{$\begin{array}{c}\text { Gene or } \\
\text { Pathway Only }\end{array}$} & \multicolumn{2}{|c|}{$\begin{array}{c}\text { Gene- or } \\
\text { Pathway-25(OH)D } \\
\text { Interaction }\end{array}$} & \multicolumn{2}{|c|}{$\begin{array}{c}\text { Gene or } \\
\text { Pathway Only }\end{array}$} & \multicolumn{2}{|c|}{$\begin{array}{c}\text { Gene- or } \\
\text { Pathway-25(OH)D } \\
\text { Interaction }\end{array}$} & \multicolumn{2}{|c|}{$\begin{array}{c}\text { Gene or } \\
\text { Pathway Only }\end{array}$} & \multicolumn{2}{|c|}{$\begin{array}{c}\text { Gene- or } \\
\text { Pathway-25(OH)D } \\
\text { Interaction }\end{array}$} \\
\hline & & & $P$ & $P_{B H}{ }^{a}$ & $P$ & $P_{B H}$ & $P$ & $P_{B H}$ & $P$ & $P_{B H}$ & $P$ & $P_{B H}$ & $P$ & $P_{B H}$ \\
\hline Cell Cycle & 60 & 56 & 0.120 & & 0.852 & & 0.030 & & 0.845 & & 0.482 & & 0.916 & \\
\hline KRAS & 13 & 13 & 0.511 & 0.777 & 0.498 & 0.999 & 0.262 & 0.815 & 0.436 & 0.990 & 0.182 & 0.737 & 0.925 & 0.993 \\
\hline FOS (c-fos) & 10 & 9 & 0.389 & 0.777 & 0.425 & 0.999 & 0.230 & 0.815 & 0.518 & 0.990 & 0.764 & 0.967 & 0.574 & 0.991 \\
\hline JUN & 7 & 7 & 0.569 & 0.784 & 0.407 & 0.999 & 0.411 & 0.815 & 0.163 & 0.770 & 0.917 & 0.967 & 0.811 & 0.991 \\
\hline C-MYC region ${ }^{d}$ & 13 & 13 & 0.010 & 0.295 & 0.843 & 0.999 & 0.051 & 0.647 & 0.857 & 0.990 & 0.054 & 0.459 & 0.959 & 0.993 \\
\hline CCND1 & 4 & 3 & 0.177 & 0.741 & 0.893 & 0.999 & 0.155 & 0.657 & 0.565 & 0.990 & 0.549 & 0.944 & 0.763 & 0.991 \\
\hline BCL2A1 & 2 & 2 & 0.118 & 0.741 & 0.587 & 0.999 & 0.083 & 0.647 & 0.574 & 0.990 & 0.250 & 0.747 & 0.915 & 0.993 \\
\hline BAX & 6 & 5 & 0.308 & 0.777 & 0.892 & 0.999 & 0.155 & 0.657 & 0.748 & 0.990 & 0.922 & 0.967 & 0.988 & 0.998 \\
\hline CDKN1A & 5 & 4 & 0.521 & 0.777 & 0.168 & 0.795 & 0.099 & 0.647 & 0.459 & 0.990 & 0.867 & 0.967 & 0.216 & 0.991 \\
\hline VDR binding sites & 19 & 18 & 0.036 & & 0.530 & & 0.008 & & 0.410 & & 0.256 & & 0.798 & \\
\hline VDR binding sites ${ }^{e}$ & 19 & 18 & 0.036 & 0.511 & 0.530 & 0.999 & 0.008 & 0.227 & 0.410 & 0.990 & 0.256 & 0.747 & 0.798 & 0.991 \\
\hline
\end{tabular}

${ }^{a} P$ of false discovery rate (BH; Benjamini-Hochberg or BH) method. ${ }^{b}$ rs10485165 and rs10507577 (Bejamin et al. 2007) [27]. ${ }^{c}$ rs1245541, rs4234798, rs700752, and rs780094. ${ }^{\mathrm{d}}$ Chromosome

$8 \mathrm{q} 24$ region. ${ }^{\mathrm{e}}$ SNPs located in the VDR binding sites relating to colorectal cancer and Crohn's disease risk as previously published (Ramagopalan et al. 2010) [19]. 
Table 5. Associations of season-adjusted 25(OH)D concentrations (per $24.96 \mathrm{nmol} / \mathrm{L}$ ) with CRC risk by genotypes, the EPIC study, $1992-2003$.

\begin{tabular}{|c|c|c|c|c|c|c|c|}
\hline \multirow{2}{*}{ Gene/SNP } & \multicolumn{2}{|c|}{ Major Allele Homozygotes } & \multicolumn{2}{|c|}{ Heterozygotes } & \multicolumn{2}{|c|}{ Minor Allele Homozygotes } & \multirow{2}{*}{$P_{\text {interaction }}$} \\
\hline & Cases/Controls & OR $(95 \% C I)^{a}$ & Cases/Controls & OR $(95 \% C I)^{a}$ & Cases/Controls & OR $(95 \% C I)^{a}$ & \\
\hline \multicolumn{8}{|l|}{ Vitamin D metabolism } \\
\hline CYP27B1 rs10877013 & $557 / 383$ & $1.00(0.86,1.17)$ & $510 / 311$ & $0.84(0.73,0.97)$ & $108 / 70$ & $0.61(0.40,0.93)$ & 0.024 \\
\hline CYP27B1 rs4646536 & $551 / 381$ & $1.00(0.86,1.17)$ & $485 / 300$ & $0.85(0.74,0.99)$ & $110 / 70$ & $0.62(0.41,0.95)$ & 0.034 \\
\hline GC rs1352846 & $500 / 319$ & $0.96(0.81,1.13)$ & $406 / 286$ & $0.79(0.67,0.93)$ & $104 / 68$ & $0.58(0.35,0.97)$ & 0.049 \\
\hline GC rs16846876 & $530 / 331$ & $1.05(0.90,1.23)$ & $477 / 337$ & $0.75(0.65,0.88)$ & $144 / 81$ & $0.88(0.63,1.21)$ & 0.017 \\
\hline GC rs2298850 & $584 / 384$ & $1.00(0.86,1.15)$ & $445 / 293$ & $0.80(0.68,0.94)$ & $86 / 61$ & $0.56(0.33,0.97)$ & 0.038 \\
\hline GC rs3755967 & $569 / 359$ & $0.99(0.85,1.15)$ & $436 / 297$ & $0.78(0.66,0.91)$ & $101 / 64$ & $0.66(0.40,1.09)$ & 0.034 \\
\hline GC rs842873 & $261 / 192$ & $0.73(0.58,0.91)$ & $588 / 354$ & $0.84(0.73,0.97)$ & $240 / 196$ & $1.22(0.97,1.52)$ & 0.002 \\
\hline \multicolumn{8}{|c|}{ VDR complex/Transcriptional Co-regulators and Co-activators } \\
\hline ARID1A rs11247596 & $720 / 474$ & $0.96(0.85,1.09)$ & $400 / 256$ & $0.76(0.63,0.92)$ & $53 / 34$ & $0.42(0.20,0.86)$ & 0.051 \\
\hline ARID1A rs12737946 & $998 / 640$ & $0.83(0.74,0.93)$ & $168 / 116$ & $1.25(0.95,1.66)$ & $9 / 8$ & $-\mathrm{c}$ & 0.016 \\
\hline ARID1A rs12752833 & $998 / 641$ & $0.83(0.74,0.93)$ & $165 / 114$ & $1.22(0.92,1.62)$ & $9 / 8$ & - & 0.025 \\
\hline CARM1 rs7254708 & $764 / 515$ & $0.82(0.73,0.93)$ & $208 / 144$ & $1.04(0.81,1.32)$ & $9 / 8$ & - & 0.0001 \\
\hline CHAF1A rs243341 & $596 / 403$ & $0.78(0.68,0.90)$ & $468 / 276$ & $0.95(0.80,1.13)$ & $102 / 78$ & $1.42(0.97,2.07)$ & 0.020 \\
\hline CHAF1A rs243365 & $607 / 434$ & $0.80(0.70,0.91)$ & $408 / 257$ & $1.02(0.86,1.22)$ & $50 / 44$ & $1.64(0.89,3.02)$ & 0.027 \\
\hline SMARCA2 rs2296212 & $920 / 607$ & $0.81(0.72,0.91)$ & $229 / 142$ & $1.15(0.89,1.48)$ & $14 / 7$ & $4.09(0.29,58.01)$ & 0.035 \\
\hline \multicolumn{8}{|l|}{ Inflammation } \\
\hline IL10 rs3024509 & $1015 / 657$ & $0.92(0.83,1.03)$ & $132 / 100$ & $0.62(0.45,0.85)$ & $3 / 2$ & - & 0.024 \\
\hline IL10 rs6686931 & $747 / 476$ & $0.80(0.70,0.91)$ & $373 / 243$ & $1.02(0.86,1.20)$ & $49 / 38$ & $1.42(0.59,3.39)$ & 0.029 \\
\hline
\end{tabular}

for genetic markers. ${ }^{\mathrm{c}}$ Not estimatable due to small sample size. 


\section{Discussion}

In this large European prospective case-control study nested within the EPIC cohort, we investigated whether genetic variation in the genes and pathways related to vitamin D metabolism and vitamin $\mathrm{D}$ genomic effects is associated with CRC risk, and whether these associations are modified by 25(OH)D concentrations.

We identified several genes related to vitamin D metabolism, the VDR complex, and VDR transcriptional activity associated with $25(\mathrm{OH}) \mathrm{D}$ concentrations, with an unadjusted $P<0.01$ before $\mathrm{BH}$ correction among controls. We confirmed two genes related to vitamin D metabolism, CYP27B1 and GC, and one in the VDR, which were identified in previous GWAS studies [28-32]. We also identified other genes in our study including 1) two genes that encode the transcription-related factors HDAC9 and NCOA7, involved in vitamin D transcriptional activity and VDR complex formation, and 2) two genes that encode the vitamin D-related transporters $L R P 2$ and CUBN $[33,34]$. LRP2, commonly known as megalin, is responsible for the endocytosis of the $25(\mathrm{OH}) \mathrm{D}$ vitamin D binding protein complex [35]. CUBN is an important co-receptor in the megalin-mediated endocytic pathway and patients without functioning CUBN were found to have abnormal 25(OH)D metabolism [33].

The genes associated with circulating $25(\mathrm{OH}) \mathrm{D}$ concentrations were also associated with CRC risk at unadjusted $P<0.01$ before BH correction. HDAC9 is located in a region on chromosome 7p21 [36] in which chromosomal gains were observed in primary CRC [37]. Furthermore, HDAC9 has been observed via chromatin immunoprecipitation (ChIP) assay in human osteosarcoma tissues to suppress p53 transcription and, thereby, promote cell proliferation [38]. An association of CUBN with CRC was previously reported in a meta-analysis of six GWAS studies [39], while no studies have investigated a possible association of $L R P 2$ with CRC. $L R P 2$ is expressed in multiple epithelial cell lines, including colon [35,40] and is often co-expressed with CUBN [34]. Additionally, there are no previous GWAS regarding $\mathrm{CRC}$ and $\mathrm{NCOA7}$, although Higginbotham et al., found statistically significant associations of NCOA7 gene variants with reduced breast cancer risk across three different study cohorts [41]. The NCOA7 SNPs identified in our study, however, differed from those identified by Higginbotham suggesting a possible novel CRC susceptibility locus.

Three VDR binding site SNPs were associated with CRC risk in our study population, but the associations were not statistically significant after $\mathrm{BH}$ correction. We a priori selected these VDR binding sites for genotyping based on the results of a previous study that used ChIP followed by DNA sequencing to identify 2776 VDR binding sites in lymphoblastoid cell lines treated with calcitriol, an active form of vitamin D [19]. The study found a statistically significant 4-fold increase in the enrichment of $V D R$ binding sites located in genes associated with CRC, and a 3.5-fold increase in enrichment located in genes associated with Crohn's disease [19]. Our findings suggest that genetic variation in these VDR binding sites, upregulated in response to treatment with vitamin D and relevant to colorectal carcinogenesis and inflammatory bowel diseases, may be associated with CRC risk.

TGF $\beta$ has an important role in the regulation of cell proliferation, differentiation, migration and apoptosis [42], and may be modulated by vitamin D [43]. SMAD7 and SMAD3, in the TGF $\beta$ signaling pathway, were statistically significantly associated with CRC risk after $\mathrm{BH}$ correction for multiple testing. SMAD7 SNPs were previously identified to be associated with CRC risk in several different populations [44-46] as well as in a meta-analysis of 2906 cases and 3416 controls from four previous GWAS studies [47]. SMAD7 is transcriptionally induced by cytokines from the TGF $\beta$ family and regulates the TGF $\beta$ signaling pathway via a negative feedback loop [42]; therefore, the overexpression of SMAD7 inhibits the pathway and its associated anti-neoplastic effects [42]. The active form of vitamin $\mathrm{D}$ was shown to inhibit SMAD7 in experimental models [48]. The role of SMAD3 in the development of CRC is less understood and somatic tumor mutations in this gene have been observed in only $4.3 \%$ of CRC cases [49]. SMAD3 has been identified to interact with $V D R$ and mediate a cross-talk between TGF $\beta$ and vitamin D signaling pathways [50]. An animal model found that SMAD3 may also play an important role in the TGF $\beta$ response to inflammation and bacteria-induced colon carcinogenesis [51]. Inflammation is further associated with CRC risk in our study as indicated by the interaction between 
circulating 25(OH)D concentrations, which has anti-inflammatory properties [52,53], and genetic variation in the IL10 gene encoding anti-inflammatory cytokine interleukin (IL)-10 involved in immune response to pathogens [54]. The induction of IL-10 is mediated by $1,25(\mathrm{OH})_{2} \mathrm{D}$ and is repressed with SMAD3 inhibition [48].

Chromosome $8 q 24$ polymorphisms in the cell cycle pathway were previously identified to be strongly associated with CRC risk $[47,55,56]$. However, they were not statistically significant after $\mathrm{BH}$ correction. Although the $8 q 24$ region is described as a gene desert, it is closely located to the region encoding $c-M Y C$ oncogene [57]. C-MYC controls processes related to cell growth regulation, metabolism and proliferation and is not only activated by numerous oncogenic pathways but also stimulates metabolic changes which can lead to malignant transformation [58]. Multiple studies have identified long-range physical interaction of the $8 q 24$ region with $c-M Y C$ via enhancer elements and chromatin loops $[57,59,60]$. In an experimental study, $1,25(\mathrm{OH})_{2} \mathrm{D}$ and the $V D R$ were shown to affect the c-MYC/MXD1 pathway leading to inhibition of c-MYC protein expression [61]. Using $8 q 24$ SNPs as a proxy, our results confirm an association between $c-M Y C$ and CRC risk, but do not indicate a potential modification by $25(\mathrm{OH}) \mathrm{D}$ despite a previously reported possible interaction for fatal prostate cancer risk [62].

The IGF signaling pathway plays a key role in cell growth [63]. In our study, IGF-related genetic variation was associated with rectal cancer risk at the pathway level as well as for several individual genes before BH correction. Contrary to our results, IGF genetic variants [64,65] in addition to high circulating IGF peptides [66] have been previously associated more strongly with increased colon versus rectal cancer risk.

The strengths of our study include its prospective design and high follow-up rate. The hypothesis-driven selection of pathways, genes, and SNPs, and relatively large samples size within a large cohort study allowed an extensive investigation of vitamin D-related and -responsive genetic variation and the effect modification by established biomarker of vitamin D status with CRC risk. We used the detailed data from EPIC to address potential confounding by body size and other factors; with our careful analyses suggesting no or little confounding. However, we cannot altogether discount the possibility of residual confounding nor changes in lifestyle habits between enrolment into the cohort and the eventual cancer diagnosis. Although our study was large, most interaction and stratified analyses had limited power, especially by sex and tumor location. Our power analyses (Supplementary Table S10) showed that we have sufficient power (80\%) to detect the effect associations in the range of 1.17 to 1.27 for relatively common SNPs with MAF between 40 and 10\%, respectively, using our full data set $(\mathrm{n}=1419$ matched case-control sets). In addition, most of our results were not statistically significant after BH correction for multiple testing. As to the selection of genes and pathways, we were limited by published literature on vitamin $D$ at the time of genotyping, so we may have not included all vitamin D-responsive genes. Additional experimental studies are needed to understand the biological mechanisms of the identified associations.

\section{Conclusions}

This large and comprehensive study has confirmed genetic variations in several previously identified vitamin D-related pathways associated with CRC risk in European populations, and has suggested potential new pathways related to vitamin D genomic effects and colorectal carcinogenesis.

Supplementary Materials: The following are available online at http://www.mdpi.com/2072-6643/11/8/1954/s1, Table S1: Characteristics of SNPs used in the study, Table S2: Vitamin D metabolism and transcriptional activity-related SNPs associated (unadjusted $P$-value $<0.05)$ with season-adjusted $25(\mathrm{OH}) \mathrm{D}$ concentrations among controls, the EPIC study, 1992-2003, Table S3A: Associations between SNPs in the genes involved in the vitamin D metabolism, mineral homeostasis/endocrine regulation of $1,25(\mathrm{OH}) 2 \mathrm{D}$ synthesis, and vitamin $\mathrm{D}$ transcriptional activity with season-adjusted 25(OH)D concentrations among controls, Table S3B: Associations between SNPs in the genes involved in the vitamin D metabolism, mineral homeostasis/endocrine regulation of $1,25(\mathrm{OH}) 2 \mathrm{D}$ synthesis, and vitamin D transcriptional activity with season-adjusted $25(\mathrm{OH}) \mathrm{D}$ concentrations among cases and controls combined, Table S4: Associations of SNPs with CRC risk overall and by tumor location (colon vs. rectum) using unconditional logistic regression with adjustment for age at recruitment, study center and sex, the EPIC 
study, 1992-2003, Table S5 Associations of SNPs with CRC risk overall and by tumor location (colon vs. rectum) among men and women using unconditional logistic regression with adjustment for age at recruitment, study center and sex, the EPIC study, 1992-2003, Table S6: Associations of SNPs in vitamin D-responsive genes with CRC risk overall and by tumor location (colon vs. rectum) using unconditional logistic regression with adjustment for age at recruitment, study center and sex, the EPIC study, 1992-2003, Table S7: Statistically significant associations (unadjusted $p<0.01$ ) of SNPs in vitamin D-responsive genes with CRC risk overall and by tumor location (colon vs. rectum) using unconditional logistic regression with adjustment for age at recruitment, study center and sex, the EPIC study, 1992-2003, Table S8: Associations of SNPs in vitamin D-responsive genes with CRC risk overall and by tumor location (colon vs. rectum) among men and women using unconditional logistic regression with adjustment for age at recruitment, study center and sex, the EPIC study, 1992-2003, Table S9: Associations of season-adjusted 25(OH)D concentrations (per $24.96 \mathrm{nmol} / \mathrm{L}$ ) with CRC risk by genotypes, the EPIC study, 1992-2003, Table S10: SNP-Only Minimal Detectable Effect Associations by Minor Allele Frequency for 80\% Power and $\mathrm{N}=1419$ matched cases and controls, the EPIC study, 1992-2003.

Author Contributions: Conceptualization, V.F.; Data curation, M.J.; Formal analysis, W.Z.; Funding acquisition, V.F. and M.J.; Investigation, A.S., P.F., I.R., E.R., B.B.-d.-M., F.J.B.v.D., A.T. (Anne Tjønneland), A.O., V.P., F.C., M.-C.B.-R., T.K., T.J., A.K., A.T. (Antonia Trichopoulou), P.M., D.T., S.P., V.K., C.S., G.S., E.W., S.C.-Y., N.S., A.B., M.-J.S., R.Q., P.A., B.G., S.H., A.P.-C., A.K.H., K.K.T., A.D., H.F., N.M. and M.J.G.; Project administration, V.F.; Supervision, V.F. and M.J.; Visualization, H.B.M. and W.Z.; Writing-original draft, V.F., H.B.M. and M.J.; Writing-review and editing, V.F., H.B.M., D.J.H., A.S., P.F., I.R., E.R., B.B.-d.-M., F.J.B.v.D., P.D.S., A.T. (Anne Tjønneland), A.O., V.P., F.C., M.-C.B.-R., T.K., T.J., A.K., A.T. (Antonia Trichopoulou), P.M., D.T., S.P., V.K., C.S., G.S., E.W., S.C.-Y., N.S., A.B., M.-J.S., R.Q., P.A., B.G., S.H., A.P.-C., A.K.H., K.K.T., A.D., H.F., N.M., M.J.G. and M.J.

Funding: Funding for this particular study was obtained from Wereld Kanker Onderzoek Fonds (WKOF) [Grant Number WCRF 2011-443; PI: M. Jenab], as part of the World Cancer Research Fund International grant programme. The EPIC study was supported by "Europe Against Cancer" Programme of the European Commission (SANCO); Ligue contre le Cancer; Institut Gustave Roussy; Mutuelle Générale de l'Education Nationale; Institut National de la Santé et de la Recherche Médicale (INSERM); German Cancer Aid; German Cancer Research Center; German Federal Ministry of Education and Research; Danish Cancer Society; Health Research Fund (FIS) of the Spanish Ministry of Health; the CIBER en Epidemiología y Salud Pública (CIBERESP), Spain; ISCIII RETIC (RD06/0020); Spanish Regional Governments of Andalusia, Asturias, Basque Country, Murcia (No 6236) and Navarra and the Catalan Institute of Oncology; Cancer Research UK; Medical Research Council, UK; The Hellenic Health Foundation; Italian Association for Research on Cancer; Italian National Research Council; Compagnia di San Paolo; Dutch Ministry of Public Health, Welfare and Sports (VWS), Netherlands Cancer Registry (NKR), LK Research Funds, Dutch Prevention Funds, Dutch ZON (Zorg Onderzoek Nederland), World Cancer Research Fund (WCRF), Statistics Netherlands (The Netherlands); Swedish Cancer Society; Swedish Scientific Council; Regional Governments of Skane and Vasterbotten, Sweden; and Nordforsk centre of excellence programme HELGA. Cancer Research UK (14136 to EPIC-Norfolk; C570/A16491 for EPIC-Oxford), Medical Research Council (1000143 to EPIC-Norfolk, MR/M012190/1 to EPIC-Oxford) (UK). DJH was supported by the Health Research Board of Ireland health research award HRA-PHS-2015-1142.

Acknowledgments: The authors would like to thank B. Hemon for his assistance in database preparation.

Conflicts of Interest: The authors declare no conflict of interest.

\section{Abbreviations}

CRC - colorectal cancer; MR-Mendelian randomization; RCT-randomized control trial; VDR—vitamin D receptor; 1,25(OH)2D-1,25-dihydroxyvitamin D; 25(OH)D-25-hydroxyvitamin D; SNP—single nucleotide polymorphism; EPIC-European Prospective Investigation into Cancer and Nutrition; IGF-insulin growth factor; TGF $\beta$ - tumor growth factor $\beta$; MAF-minor allele frequency; tagSNPs—tagging SNPs; BMI—body mass index; ARTP —Adaptive Rank Truncated Product; BH—Benjamini-Hochberg; ChIP—chromatin immunoprecipitation.

\section{References}

1. Ferlay, J.; Soerjomataram, I.; Dikshit, R.; Eser, S.; Mathers, C.; Rebelo, M.; Parkin, D.M.; Forman, D.; Bray, F. Cancer incidence and mortality worldwide: Sources, methods and major patterns in GLOBOCAN 2012. Int. J. Cancer 2015, 136, E359-E386. [CrossRef] [PubMed]

2. Touvier, M.; Chan, D.S.; Lau, R.; Aune, D.; Vieira, R.; Greenwood, D.C.; Kampman, E.; Riboli, E.; Hercberg, S.; Norat, T. Meta-analyses of vitamin D intake, 25-hydroxyvitamin D status, vitamin D receptor polymorphisms, and colorectal cancer risk. Cancer Epidemiol. Biomark. Prev. 2011, 20, 1003-1016. [CrossRef] [PubMed]

3. McCullough, M.L.; Zoltick, E.S.; Weinstein, S.J.; Fedirko, V.; Wang, M.; Cook, N.R.; Eliassen, A.H.; Zeleniuch-Jacquotte, A.; Agnoli, C.; Albanes, D.; et al. Circulating Vitamin D and Colorectal Cancer Risk: An International Pooling Project of 17 Cohorts. J. Natl. Cancer Inst. 2018, 111, 158-169. [CrossRef] [PubMed] 
4. Jiang, X.; O’Reilly, P.F.; Aschard, H.; Hsu, Y.-H.; Richards, J.B.; Dupuis, J.; Ingelsson, E.; Karasik, D.; Pilz, S.; Berry, D.; et al. Genome-wide association study in 79,366 European-ancestry individuals informs the genetic architecture of 25-hydroxyvitamin D levels. Nat. Commun. 2018, 9, 260. [CrossRef] [PubMed]

5. Dimitrakopoulou, V.I.; Tsilidis, K.K.; Haycock, P.C.; Dimou, N.L.; Al-Dabhani, K.; Martin, R.M.; Lewis, S.J.; Gunter, M.J.; Mondul, A.; Shui, I.M.; et al. Circulating vitamin D concentration and risk of seven cancers: Mendelian randomisation study. BMJ 2017, 359, j4761. [CrossRef] [PubMed]

6. He, Y.; Timofeeva, M.; Farrington, S.M.; Vaughan-Shaw, P.; Svinti, V.; Walker, M.; Zgaga, L.; Meng, X.; Li, X.; Spiliopoulou, A.; et al. Exploring causality in the association between circulating 25-hydroxyvitamin D and colorectal cancer risk: A large Mendelian randomisation study. BMC Med. 2018, 16, 142. [CrossRef]

7. Wactawski-Wende, J.; Kotchen, J.M.; Anderson, G.L.; Assaf, A.R.; Brunner, R.L.; O'Sullivan, M.J.; Margolis, K.L.; Ockene, J.K.; Phillips, L.; Pottern, L.; et al. Calcium plus vitamin D supplementation and the risk of colorectal cancer. N. Engl. J. Med. 2006, 354, 684-696. [CrossRef]

8. Baron, J.A.; Barry, E.L.; Mott, L.A.; Rees, J.R.; Sandler, R.S.; Snover, D.C.; Bostick, R.M.; Ivanova, A.; Cole, B.F.; Ahnen, D.J.; et al. A Trial of Calcium and Vitamin D for the Prevention of Colorectal Adenomas. N. Engl. J. Med. 2015, 373, 1519-1530. [CrossRef]

9. Manson, J.E.; Cook, N.R.; Lee, I.M.; Christen, W.; Bassuk, S.S.; Mora, S.; Gibson, H.; Gordon, D.; Copeland, T.; D'Agostino, D.; et al. Vitamin D Supplements and Prevention of Cancer and Cardiovascular Disease. $N$. Engl. J. Med. 2018, 380, 33-44. [CrossRef]

10. Barry, E.L.; Rees, J.R.; Peacock, J.L.; Mott, L.A.; Amos, C.I.; Bostick, R.M.; Figueiredo, J.C.; Ahnen, D.J.; Bresalier, R.S.; Burke, C.A.; et al. Genetic Variants in CYP2R1, CYP24A1, and VDR Modify the Efficacy of Vitamin D3 Supplementation for Increasing Serum 25-Hydroxyvitamin D Levels in a Randomized Controlled Trial. J. Clin. Endocrinol. Metab. 2014, 99, E2133-E2137. [CrossRef]

11. Matusiak, D.; Murillo, G.; Carroll, R.E.; Mehta, R.G.; Benya, R.V. Expression of vitamin D receptor and 25-hydroxyvitamin D3-1 $\alpha$-hydroxylase in normal and malignant human colon. Cancer Epidemiol. Biomark. Prev. 2005, 14, 2370-2376. [CrossRef] [PubMed]

12. Tangpricha, V.; Flanagan, J.N.; Whitlatch, L.W.; Tseng, C.C.; Chen, T.C.; Holt, P.R.; Lipkin, M.S.; Holick, M.F. 25-hydroxyvitamin D-1alpha-hydroxylase in normal and malignant colon tissue. Lancet 2001, 357, 1673-1674. [CrossRef]

13. Matusiak, D.; Benya, R.V. CYP27A1 and CYP24 expression as a function of malignant transformation in the colon. J. Histochem. Cytochem. 2007, 55, 1257-1264. [CrossRef] [PubMed]

14. Ball, G.F.M. Vitamins: Their Role in the Human Body; Blackwell Science: Ames, IA, USA, 2004.

15. Feldman, D.; Krishnan, A.V.; Swami, S.; Giovannucci, E.; Feldman, B.J. The role of vitamin D in reducing cancer risk and progression. Nat. Rev. Cancer 2014, 14, 342-357. [CrossRef] [PubMed]

16. Slatter, M.L.; Yakumo, K.; Hoffman, M.; Neuhausen, S. Variants of the VDR gene and risk of colon cancer (United States). Cancer Causes Control 2001, 12, 359-364. [CrossRef] [PubMed]

17. Poynter, J.N.; Jacobs, E.T.; Figueiredo, J.C.; Lee, W.H.; Conti, D.V.; Campbell, P.T.; Levine, A.J.; Limburg, P.; Le Marchand, L.; Cotterchio, M.; et al. Genetic variation in the vitamin D receptor (VDR) and the vitamin D-binding protein (GC) and risk for colorectal cancer: Results from the Colon Cancer Family Registry. Cancer Epidemiol. Biomark. Prev. 2010, 19, 525-536. [CrossRef] [PubMed]

18. Gibbs, D.C.; Fedirko, V.; Um, C.; Gross, M.D.; Thyagarajan, B.; Bostick, R.M. Associations of Circulating 25-Hydroxyvitamin D3 Concentrations With Incident, Sporadic Colorectal Adenoma Risk According to Common Vitamin D-Binding Protein Isoforms. Am. J. Epidemiol. 2018, 187, 1923-1930. [CrossRef]

19. Ramagopalan, S.V.; Heger, A.; Berlanga, A.J.; Maugeri, N.J.; Lincoln, M.R.; Burrell, A.; Handunnetthi, L.; Handel, A.E.; Disanto, G.; Orton, S.M.; et al. A ChIP-seq defined genome-wide map of vitamin D receptor binding: Associations with disease and evolution. Genome Res. 2010, 20, 1352-1360. [CrossRef]

20. Gil, A.; Plaza-Diaz, J.; Mesa, M.D. Vitamin D: Classic and Novel Actions. Ann. Nutr. Metab. 2018, 72, 87-95. [CrossRef]

21. Riboli, E.; Hunt, K.J.; Slimani, N.; Ferrari, P.; Norat, T.; Fahey, M.; Charrondiere, U.R.; Hemon, B.; Casagrande, C.; Vignat, J.; et al. European Prospective Investigation into Cancer and Nutrition (EPIC): Study populations and data collection. Public Health Nutr. 2002, 5, 1113-1124. [CrossRef]

22. Bingham, S.; Riboli, E. Diet and cancer-the European Prospective Investigation into Cancer and Nutrition. Nat. Rev. Cancer 2004, 4, 206-215. [CrossRef] [PubMed] 
23. Jenab, M.; Bueno-de-Mesquita, H.B.; Ferrari, P.; van Duijnhoven, F.J.; Norat, T.; Pischon, T.; Jansen, E.H.; Slimani, N.; Byrnes, G.; Rinaldi, S.; et al. Association between pre-diagnostic circulating vitamin D concentration and risk of colorectal cancer in European populations:a nested case-control study. BMJ 2010, 340, b5500. [CrossRef] [PubMed]

24. Gail, M.H.; Wu, J.; Wang, M.; Yaun, S.S.; Cook, N.R.; Eliassen, A.H.; McCullough, M.L.; Yu, K.; Zeleniuch-Jacquotte, A.; Smith-Warner, S.A.; et al. Calibration and seasonal adjustment for matched case-control studies of vitamin D and cancer. Stat. Med. 2016, 35, 2133-2148. [CrossRef] [PubMed]

25. Yu, K.; Li, Q.; Bergen, A.W.; Pfeiffer, R.M.; Rosenberg, P.S.; Caporaso, N.; Kraft, P.; Chatterjee, N. Pathway analysis by adaptive combination of P-values. Genet. Epidemiol. 2009, 33, 700-709. [CrossRef] [PubMed]

26. Benjamini, Y.; Hochberg, Y. Controlling the False Discovery Rate-A Practical and Powerful Approach to Multiple Testing. J. Roy. Stat. Soc. B Methodol. 1995, 57, 289-300. [CrossRef]

27. Benjamin, E.J.; Dupuis, J.; Larson, M.G.; Lunetta, K.L.; Booth, S.L.; Govindaraju, D.R.; Kathiresan, S.; Keaney, J.F., Jr.; Keyes, M.J.; Lin, J.P.; et al. Genome-wide association with select biomarker traits in the Framingham Heart Study. BMC Med. Genet. 2007, 8 (Suppl. 1), S11. [CrossRef] [PubMed]

28. Hibler, E.A.; Klimentidis, Y.C.; Jurutka, P.W.; Kohler, L.N.; Lance, P.; Roe, D.J.; Thompson, P.A.; Jacobs, E.T. CYP24A1 and CYP27B1 Polymorphisms, Concentrations of Vitamin D Metabolites, and Odds of Colorectal Adenoma Recurrence. Nutr. Cancer 2015, 67, 1131-1141. [CrossRef]

29. Ahn, J.; Albanes, D.; Berndt, S.I.; Peters, U.; Chatterjee, N.; Freedman, N.D.; Abnet, C.C.; Huang, W.-Y.; Kibel, A.S.; Crawford, E.D.; et al. Vitamin D-related genes, serum vitamin D concentrations and prostate cancer risk. Carcinogenesis 2009, 30, 769-776. [CrossRef]

30. Wang, T.J.; Zhang, F.; Richards, J.B.; Kestenbaum, B.; van Meurs, J.B.; Berry, D.; Kiel, D.; Streeten, E.A.; Ohlsson, C.; Koller, D.L.; et al. Common genetic determinants of vitamin D insufficiency: A genome-wide association study. Lancet 2010, 376, 180-188. [CrossRef]

31. Bu, F.X.; Armas, L.; Lappe, J.; Zhou, Y.; Gao, G.; Wang, H.W.; Recker, R.; Zhao, L.J. Comprehensive association analysis of nine candidate genes with serum 25-hydroxy vitamin D levels among healthy Caucasian subjects. Hum. Genet. 2010, 128, 549-556. [CrossRef]

32. Mokry, L.E.; Ross, S.; Ahmad, O.S.; Forgetta, V.; Smith, G.D.; Goltzman, D.; Leong, A.; Greenwood, C.M.; Thanassoulis, G.; Richards, J.B. Vitamin D and Risk of Multiple Sclerosis: A Mendelian Randomization Study. PLoS Med. 2015, 12, e1001866. [CrossRef] [PubMed]

33. Nykjaer, A.; Fyfe, J.C.; Kozyraki, R.; Leheste, J.-R.; Jacobsen, C.; Nielsen, M.S.; Verroust, P.J.; Aminoff, M.; de la Chapelle, A.; Moestrup, S.K.; et al. Cubilin dysfunction causes abnormal metabolism of the steroid hormone 25(OH) vitamin D3. Proc. Natl. Acad. Sci. USA 2001, 98, 13895-13900. [CrossRef] [PubMed]

34. Kaseda, R.; Hosojima, M.; Sato, H.; Saito, A. Role of megalin and cubilin in the metabolism of vitamin $\mathrm{D}(3)$. Ther. Apher. Dial. 2011, 15 (Suppl. 1), 14-17. [CrossRef] [PubMed]

35. Rowling, M.J.; Kemmis, C.M.; Taffany, D.A.; Welsh, J. Megalin-Mediated Endocytosis of Vitamin D Binding Protein Correlates with 25-Hydroxycholecalciferol Actions in Human Mammary Cells. J. Nutr. 2006, 136, 2754-2759. [CrossRef] [PubMed]

36. Mahlknecht, U.; Schnittger, S.; Will, J.; Cicek, N.; Hoelzer, D. Chromosomal organization and localization of the human histone deacetylase 9 gene (HDAC9). Biochem. Biophys. Res. Commun. 2002, 293, 182-191. [CrossRef]

37. Aragane, H.; Sakakura, C.; Nakanishi, M.; Yasuoka, R.; Fujita, Y.; Taniguchi, H.; Hagiwara, A.; Yamaguchi, T.; Abe, T.; Inazawa, J.; et al. Chromosomal aberrations in colorectal cancers and liver metastases analyzed by comparative genomic hybridization. Int. J. Cancer 2001, 94, 623-629. [CrossRef] [PubMed]

38. Zhao, Y.-X.; Wang, Y.-S.; Cai, Q.-Q.; Wang, J.-Q.; Yao, W.-T. Up-regulation of HDAC9 promotes cell proliferation through suppressing p53 transcription in osteosarcoma. Int. J. Clin. Exp. Med. 2015, 8, 11818-11823. [PubMed]

39. Al-Tassan, N.A.; Whiffin, N.; Hosking, F.J.; Palles, C.; Farrington, S.M.; Dobbins, S.E.; Harris, R.; Gorman, M.; Tenesa, A.; Meyer, B.F.; et al. A new GWAS and meta-analysis with 1000Genomes imputation identifies novel risk variants for colorectal cancer. Sci. Rep. 2015, 5, 10442. [CrossRef] [PubMed]

40. Ternes, S.B.; Rowling, M.J. Vitamin D Transport Proteins Megalin and Disabled-2 Are Expressed in Prostate and Colon Epithelial Cells and Are Induced and Activated by All-Trans-Retinoic Acid. Nutr. Cancer 2013, 65, 900-907. [CrossRef] 
41. Higginbotham, K.S.P.; Breyer, J.P.; Bradley, K.M.; Schuyler, P.A.; Plummer, W.D.; Freudenthal, M.E.; Trentham-Dietz, A.; Newcomb, P.A.; Sanders, M.E.; Page, D.L.; et al. A multi-stage association study identifies a breast cancer genetic locus at NCOA7. Cancer Res. 2011, 71, 3881-3888. [CrossRef]

42. Yan, X.; Liu, Z.; Chen, Y. Regulation of TGF-beta signaling by Smad7. Acta Biochim. Biophys. Sin. 2009, 41, 263-272. [CrossRef] [PubMed]

43. Tu, H.; Flanders, W.D.; Ahearn, T.U.; Daniel, C.R.; Gonzalez-Feliciano, A.G.; Long, Q.; Rutherford, R.E.; Bostick, R.M. Effects of Calcium and Vitamin D(3) on Transforming Growth Factors in Rectal Mucosa of Sporadic Colorectal Adenoma Patients: A Randomized Controlled Trial. Mol. Carcinog. 2015, 54, 270-280. [CrossRef] [PubMed]

44. Jiang, X.; Castelao, J.E.; Vandenberg, D.; Carracedo, A.; Redondo, C.M.; Conti, D.V.; Paredes Cotore, J.P.; Potter, J.D.; Newcomb, P.A.; Passarelli, M.N.; et al. Genetic variations in SMAD7 are associated with colorectal cancer risk in the colon cancer family registry. PLoS ONE 2013, 8, e60464. [CrossRef] [PubMed]

45. Zhang, B.; Jia, W.H.; Matsuo, K.; Shin, A.; Xiang, Y.B.; Matsuda, K.; Jee, S.H.; Kim, D.H.; Cheah, P.Y.; Ren, Z.; et al. Genome-wide association study identifies a new SMAD7 risk variant associated with colorectal cancer risk in East Asians. Int. J. Cancer 2014, 135, 948-955. [CrossRef] [PubMed]

46. Kirac, I.; Matosevic, P.; Augustin, G.; Simunovic, I.; Hostic, V.; Zupancic, S.; Hayward, C.; Antoljak, N.; Rudan, I.; Campbell, H.; et al. SMAD7 variant rs4939827 is associated with colorectal cancer risk in Croatian population. PLoS ONE 2013, 8, e74042. [CrossRef] [PubMed]

47. Peters, U.; Hutter, C.M.; Hsu, L.; Schumacher, F.R.; Conti, D.V.; Carlson, C.S.; Edlund, C.K.; Haile, R.W.; Gallinger, S.; Zanke, B.W.; et al. Meta-analysis of New Genome-wide Association Studies of Colorectal Cancer Risk. Hum. Genet. 2012, 131, 217-234. [CrossRef] [PubMed]

48. Nanduri, R.; Mahajan, S.; Bhagyaraj, E.; Sethi, K.; Kalra, R.; Chandra, V.; Gupta, P. The Active Form of Vitamin D Transcriptionally Represses Smad7 Signaling and Activates Extracellular Signal-regulated Kinase (ERK) to Inhibit the Differentiation of a Inflammatory T Helper Cell Subset and Suppress Experimental Autoimmune Encephalomyelitis. J. Biol. Chem. 2015, 290, 12222-12236. [CrossRef] [PubMed]

49. Fleming, N.I.; Jorissen, R.N.; Mouradov, D.; Christie, M.; Sakthianandeswaren, A.; Palmieri, M.; Day, F.; Li, S.; Tsui, C.; Lipton, L.; et al. SMAD2, SMAD3 and SMAD4 mutations in colorectal cancer. Cancer Res. 2013, 73, 725-735. [CrossRef] [PubMed]

50. Yanagisawa, J.; Yanagi, Y.; Masuhiro, Y.; Suzawa, M.; Watanabe, M.; Kashiwagi, K.; Toriyabe, T.; Kawabata, M.; Miyazono, K.; Kato, S. Convergence of transforming growth factor-beta and vitamin D signaling pathways on SMAD transcriptional coactivators. Science 1999, 283, 1317-1321. [CrossRef]

51. Maggio-Price, L.; Treuting, P.; Zeng, W.; Tsang, M.; Bielefeldt-Ohmann, H.; Iritani, B.M. Helicobacter infection is required for inflammation and colon cancer in SMAD3-deficient mice. Cancer Res. 2006, 66, 828-838. [CrossRef]

52. Lamprecht, S.A.; Lipkin, M. Chemoprevention of colon cancer by calcium, vitamin D and folate: Molecular mechanisms. Nat. Rev. Cancer 2003, 3, 601-614. [CrossRef] [PubMed]

53. Bostick, R.M.; Goodman, M.; Sidelnikov, E. Calcium and vitamin D. In Genetics of Colorectal Cancer; Potter, J.D., Lindor, N.M., Eds.; Springer Science + Business Media, LLC: New York, NY, USA, 2009; pp. 277-296.

54. Iyer, S.S.; Cheng, G. Role of Interleukin 10 Transcriptional Regulation in Inflammation and Autoimmune Disease. Crit. Rev. Immunol. 2012, 32, 23-63. [CrossRef] [PubMed]

55. Hutter, C.M.; Slattery, M.L.; Duggan, D.J.; Muehling, J.; Curtin, K.; Hsu, L.; Beresford, S.A.; Rajkovic, A.; Sarto, G.E.; Marshall, J.R.; et al. Characterization of the association between 8q24 and colon cancer: Gene-environment exploration and meta-analysis. BMC Cancer 2010, 10, 670. [CrossRef] [PubMed]

56. Wang, Y.P.; Zhang, J.; Zhu, H.Y.; Qian, C.L.; Liu, H.; Ji, F.; Shen, Z.Y. Common variation rs6983267 at 8 q24.1 and risk of colorectal adenoma and cancer: Evidence based on 31 studies. Tumour Biol. 2014, 35, 4067-4075. [CrossRef] [PubMed]

57. Pomerantz, M.M.; Ahmadiyeh, N.; Jia, L.; Herman, P.; Verzi, M.P.; Doddapaneni, H.; Beckwith, C.A.; Chan, J.A.; Hills, A.; Davis, M.; et al. The 8q24 cancer risk variant rs6983267 demonstrates long-range interaction with MYC in colorectal cancer. Nat. Genet. 2009, 41, 882-884. [CrossRef] [PubMed]

58. Miller, D.M.; Thomas, S.D.; Islam, A.; Muench, D.; Sedoris, K. c-Myc and Cancer Metabolism. Clin. Cancer Res. 2012, 18, 5546-5553. [CrossRef] [PubMed] 
59. Ahmadiyeh, N.; Pomerantz, M.M.; Grisanzio, C.; Herman, P.; Jia, L.; Almendro, V.; He, H.H.; Brown, M.; Liu, X.S.; Davis, M.; et al. 8q24 prostate, breast, and colon cancer risk loci show tissue-specific long-range interaction with MYC. Proc. Natl. Acad. Sci. USA 2010, 107, 9742-9746. [CrossRef] [PubMed]

60. Wright, J.B.; Brown, S.J.; Cole, M.D. Upregulation of c-MYC in cis through a Large Chromatin Loop Linked to a Cancer Risk-Associated Single-Nucleotide Polymorphism in Colorectal Cancer Cells. Mol. Cell. Biol. 2010, 30, 1411-1420. [CrossRef]

61. Salehi-Tabar, R.; Nguyen-Yamamoto, L.; Tavera-Mendoza, L.E.; Quail, T.; Dimitrov, V.; An, B.-S.; Glass, L.; Goltzman, D.; White, J.H. Vitamin D receptor as a master regulator of the c-MYC/MXD1 network. Proc. Natl. Acad. Sci. USA 2012, 109, 18827-18832. [CrossRef]

62. Dimitrakopoulou, V.I.; Travis, R.C.; Shui, I.M.; Mondul, A.; Albanes, D.; Virtamo, J.; Agudo, A.; Boeing, H.; Bueno-de-Mesquita, H.B.; Gunter, M.J.; et al. Interactions Between Genome-Wide Significant Genetic Variants and Circulating Concentrations of 25-Hydroxyvitamin D in Relation to Prostate Cancer Risk in the National Cancer Institute BPC3. Am. J. Epidemiol. 2017, 185, 452-464. [CrossRef]

63. Vigneri, P.G.; Tirrò, E.; Pennisi, M.S.; Massimino, M.; Stella, S.; Romano, C.; Manzella, L. The Insulin/IGF System in Colorectal Cancer Development and Resistance to Therapy. Front. Oncol. 2015, 5, 230. [CrossRef] [PubMed]

64. Wong, H.L.; Delellis, K.; Probst-Hensch, N.; Koh, W.P.; Van Den Berg, D.; Lee, H.P.; Yu, M.C.; Ingles, S.A. A new single nucleotide polymorphism in the insulin-like growth factor I regulatory region associates with colorectal cancer risk in singapore chinese. Cancer Epidemiol. Biomark. Prev. 2005, 14, 144-151.

65. Simons, C.C.J.M.; Schouten, L.J.; Godschalk, R.W.L.; van Engeland, M.; van den Brandt, P.A.; van Schooten, F.J.; Weijenberg, M.P. Genetic Variants in the Insulin-like Growth Factor Pathway and Colorectal Cancer Risk in the Netherlands Cohort Study. Sci. Rep. 2015, 5, 14126. [CrossRef] [PubMed]

66. Chi, F.; Wu, R.; Zeng, Y.C.; Xing, R.; Liu, Y. Circulation insulin-like growth factor peptides and colorectal cancer risk: An updated systematic review and meta-analysis. Mol. Biol. Rep. 2013, 40, 3583-3590. [CrossRef] [PubMed]

(C) 2019 by the authors. Licensee MDPI, Basel, Switzerland. This article is an open access article distributed under the terms and conditions of the Creative Commons Attribution (CC BY) license (http://creativecommons.org/licenses/by/4.0/). 